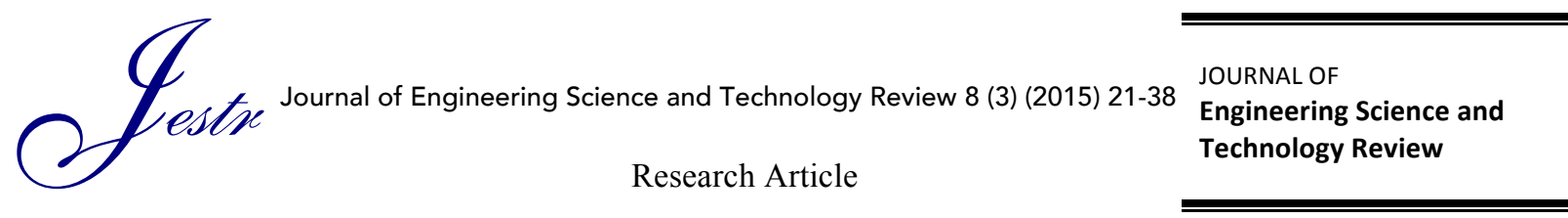

www.jestr.org

\title{
Anatomy of the Pulsating Double Layer Source in the Earth's Magnetotail
}

\author{
D. V. Sarafopoulos* \\ Department of Electrical and Computer Engineering, Democritus University of Thrace, Xanthi, Greece.
}

Received 2 October 2014; Accepted 13 July 2015

\begin{abstract}
This investigation is composed of an observational part, plus a new theoretical model interpreting the related exhibited satellite datasets through an entirely new approach concerning the substorm's ultimate excitation mechanism. First, we present a few representative case studies showing the $B_{z}$ component of the magnetic field to develop quasi-periodic negative deflections with periodicities ranging from $\mathrm{T}=15$ to $60 \mathrm{~s}$ in the central plasma sheet (CPS) with persistent tailward plasma flows. The wave activity of $\mathrm{B}_{z}$ is much lower outside the CPS, while occasionally almost a monochromatic response is identified. In certain cases, a profound change of frequency is evident and happens most probably due to the Doppler-effect; there is a relation between the plasma velocity enhancement and the increase of frequency. When comparing the amplitude of Bz deflections to the lobe magnetic field, one plausibly may infer that the deflections reflect the very dynamics of the source itself. Therefore, we put forward the concept about "a pulsating source", and more specifically, we adopt the pulsating "twin Double Layer (DL) structure" as the ultimate mechanism converting magnetic energy to kinetic. The DL acts either as a resistor or a capacitor and the twin-DL structure approximately behaves in a way similar to the cardiac cycle with repeated, rhythmic contractions and expansions. The contraction phase represents the abrupt local thinning of the plasma sheet (PS) that terminates explosively, heating the plasma and accelerating energetic particles. The expansion phase corresponds to a local distention of the source, which also terminates explosively. The DLs are oppositely polarized depending on the range of Rc whether it fulfills the condition $r_{g i} \leq R_{c}<9 r_{g i}$ or $r_{g e}<R_{c}<r_{g i}$, where $r_{g i}\left(r_{g e}\right)$ and $R_{c}$ are the ion (electron) gyro-radius and the curvature radius of the local magnetic field, respectively. In more detail, the "akis structure", as it was earlier introduced by Sarafopoulos $[1,2]$ and further elaborated in this work, repetitively forms DLs that undergo a transition from depolarization to repolarization in each of the consecutive cycles. The collapse of the dissipation region (leading to local dipolarization) seems to occur when $r_{\text {ge }}<\mathrm{Rc}<9 \mathrm{r}_{\text {ge. }}$. Large scale Boström's type II field aligned currents (FACs) flow and neutralize the net charges produced by "charge separation processes" within the twin-DL structure; thus the whole structure principally operates (under favorable conditions) like a huge resonant RLC-type circuit. Actually the pulsating source is an integral part of that huge (resonating) circuit, wherein even the ionosphere obviously plays its vital role imposing a positive feedback. The author of this study believes that Faraday's law can be experimentally validated with his suggested model more reliably than with the alternative X-type magnetic reconnection model incorporating the concept of "magnetic diffusion". The presently proposed "akis structure" embedded in the whole magnetotail's current system is presumably a precious structure with novel features. The source will probably be triggered at that place in which the condition for resonance is satisfied, while the resonance dramatically increases the rate of energy conversion, that is the efficiency of the source.
\end{abstract}

Keywords: Plasma sheet, magnetic reconnection, double layers, field-aligned currents, pulsating source, plasma instabilities.

\section{Introduction}

In the past, the idea of "multiple and turbulent reconnection" in plasma sheet (PS) was commonly used (in published literature), but the concept about a systematically and wellorganized "pulsating source", that is a source being characterized by repeated, rhythmic contractions and expansions, has never been seriously proposed and examined; perhaps (partially) because the current reconnection models are mostly quasi-stationary with respect to time. Certainly, by the term "pulsating source", we

* E-mail address: $f$ sarafo@ee.duth.gr ISSN: 1791-2377 @ 2015 Kavala Institute of Technology. All rights reserved. mean a well developed dissipation region with its own inherent dynamics, which, in parallel, is embedded in the whole system-circuit that occasionally is excited to large scale resonances. Apparently in the latter case more efficient plasma heating and high-energy particle accelerations probably take place. In this perspective, the source will be cyclically activated having a natural resonance frequency; and the vibrations would grow to very large amplitudes and be clearly identified in the magnetotail. Eventually, in the context of this work, taking into account the disparity of the small dissipation region in relation to the large scale system (extended all the way from the source inside the tail to the 
ionosphere), a precious diagnostic tool grasping the inner nature, topology and dynamics of the source itself could be derived.

In the observational part of this work, we present a few indicative case studies demonstrating a profound quasiperiodic response visible along the $\mathrm{B}_{\mathrm{z}}$-trace of the magnetic field in central plasma sheet (CPS) and during periods of intense tailward plasma flows. Under these conditions, the minima for the $B_{z}$ deflections are characterized by a negative sign; while the $B_{z}$ averages out to negative values, too. Certainly, these very well-organized and substormassociated fluctuations are of supreme importance, as far as the magnetotail's dynamics is considered; and for this reason, we put forward the concept for a pulsating source whose periodicities range from 15 to $60 \mathrm{~s}$. Furthermore, since the negative $B_{z}$ fluctuations or the overall $\Delta B_{z}$ transitions in CPS reach values comparable to the lobe magnetic field magnitude, one may further assume that the source indeed reveals a natural resonance frequency corresponding to repetitive activations or even cross-tail current disruptions (CDs).

Then we try to establish the fundamental physics for all the involved processes justifying the assumption of a resonating source. It is unavoidable that such a response is probably the synthesis result of local and large- or even global-scale processes in the magnetosphere. The physics of local and thin current sheets (TCSs) in the magnetotail must couple to the ionosphere response via the very large scale field-aligned currents (FACs). The whole circuit seems to develop a unique natural resonance frequency depending on the time; consequently, all the circuit elements have to vary unceasingly (from time to time). Most importantly, we attempt to obtain an insight of the source's interior structure and functionality. Thus, in the next section, given that the suggested source recurrently contracts and expands, we shall exhibit a more detailed picture concerning these two distinct source phases (integrating functional and geometrical elements alike). More specifically to this direction, we use as a source model the "twin-DL structure", which was first suggested by Sarafopoulos [2]. However, this initial model is radically elaborated here; now, for the first time, we propose the whole procedure of source evolution leading to a cyclic mode composed of two alternating and distinct phases. In the consecutive activations of the source, the twin-DL structure seems to be the promising "current pump" developed in very thin current sheets. This version of the model may offer a fresh look into the magnetotail's dynamics as a whole. It would be meaningless to begin an unceasing meditation about the "X-type reconnection model" as compared to the "twin-DL structure". Many morphological features and functionality aspects certainly remain the same, but the whole philosophy is different: The DLs directly convert the stored magnetic energy (within the distributed inductance of the circuit) into plasma kinetic energy; the latter occurs instantaneously, at the interior of a DL, whenever the circuit's current varies abruptly (halts). The produced electromotive force (emf) is defined by the relation $\mathrm{V}=\mathrm{L} \cdot \mathrm{d} \mathrm{I} / \mathrm{dt}$, where $\mathrm{L}$ denotes the inductance of the equivalent circuit. Thus, there is a confidence that Faraday's law is directly implemented. In our approach, the magnetotail/ionosphere circuit associated with the source mimics, in a large degree, the response of an RLC circuit, where $\mathrm{R}$ is the resistor, $\mathrm{L}$ the inductor and $\mathrm{C}$ the capacitor of the huge circuit. The circuit element DL behaves either as a capacitor or as resistor; it alternates its function within each variation cycle. Obviously, in this perspective the displacement current plays its vital role; and the whole approach has nothing to do with any MHD treatment. The key process for the functionality of the twin-DL entity is not only a charge separation process taking place inside, but additional procedures leading to charge redistributions in each variation cycle. The source's contraction/expansion phases seem to correspond to a depolarization/repolarization cycle in each $D L$. Finally, as a result, the charge redistribution reverses the direction of current flow in the whole circuit; a process comparable to what occurs in a resonating $\mathrm{LC}$ circuit, for each cycle. The twin-DL structure pulsates with periods ranging from $\mathrm{T}=15$ to $60 \mathrm{~s}$; although the recorded period sometimes seems to be longer. The reason for the latter is that, as we shall infer on the basis of our events, the measured frequency is strongly affected from the plasma velocity; that is, the "Doppler effect" significantly modifies the source's frequency. And from this perspective, the waves associated with higher plasma velocities probably give a better estimate for the authentic (real) value of the source's resonance frequency; we suppose that higher velocities occur closer to the source, since the PS is supposed thinner near the source. Nevertheless, the author of this work, based on his previous research efforts and experience, has come to the conclusion that the above typical values of $\mathrm{T}$ are sometimes extended to even lower or higher ranges.

Furthermore, on the basis of the suggested pulsating twin-DL structure, a satisfactory answer is probably given upon an open question concerning the electron acceleration mechanism in the magnetotail. It seems to be a mystery that the energetic electrons in magnetotail always form a bi-layer structure with counterstreaming fluxes, parallel and antiparallel to the local ambient magnetic field lines, as it was established by Sarafopoulos [3]. In particular, he stressed that the tailward directed fluxes always occur in a region adjacent to the lobes; all these issues are extensively scrutinized in the discussion section.

A few works in the past, relevant to the idea about a "pulsating source", can not escape our attention; thus, we would like to emphasize the following research efforts:

\section{1]. The "IVD effect" of energetic particles}

For many years there is a worth mentioning evidence that the acceleration source in magnetotail is inherently associated with a repetition mode or even a natural resonance frequency: In 1988, fine-time resolution (10 s) observations of bursts of energetic particles inside the Earth's plasma sheet (PS), by the IMP-8 spacecraft, were reported by Sarafopoulos and Sarris [4]. They discovered that the detailed structure of the time-intensity profiles of the ion bursts often display the "inverse velocity dispersion" (IVD) effect, whereby the low energy $(\sim 300 \mathrm{keV})$ ion intensity enhancements are detected 10-20 s before the higher energy $(1 \mathrm{MeV})$ ones. In particular, it was found that long duration (several minutes or more) bursts of energetic particles are composed of a series of highly anisotropic, short duration impulsive bursts, which last a few tens of seconds, showing the IVD effect. Furthermore, since the IVD effect is observed (a) both inside the plasma sheet and adjacent to the lobes, (b) during periods of thinning as well as expansion of the plasma sheet, and (c) for both tailward and earthward streaming of the energetic ions; it is interpreted as the growth time of the source itself. In this perspective the IVD is assumed being the fingerprint of the source itself, and given that these bursts are characterized by a repetition mode, one could infer that actually in 
magnetotail the source might be pulsating. From the presented events (examined by Sarafopoulos and Sarris) one can indeed infer that usually the time in between two successive IVDs is 40-60 s. Certainly, such a repetition time corresponds to the upper band of periodicities assumed here (i.e., $\mathrm{T}=15-60 \mathrm{~s}$ ); however, higher repetition rates for the IVD events could not be recognized on the basis of IMP-8 available time resolutions.

\section{2]. An event with quasi-periodic current disruptions}

One more work directly related to our purpose is that of Takahashi et al. [5], in which a particularly important substorm event was studied, which occurred at a radial distance of $\sim 8 \mathrm{R}_{\mathrm{E}}$, displaying large amplitude (10-40 nT) oscillations of the total field with a period of $\sim 13 \mathrm{~s}$ and southward turning of the field during most oscillation cycles. They assumed that the detected dipolarization means that the spacecraft made an in-situ observation of the disruption of the cross tail current sheet. They did not clearly suggest a pulsating source being at work; however, they wrote that "we might have tearing islands that are convected or created in a periodic fashion". Most importantly, they speculated (among other things) that the periodicity of $13 \mathrm{~s}$ may be related to the bounce period $(\sim 13 \mathrm{~s})$ of $0.5 \mathrm{keV}$ electrons at $\mathrm{L}=8$. Such a hypothesis seems to be very close to our suggested model, adopting, in a large degree, the behavior of an LC circuit, in which the charge flows earthward and tailward in each variation cycle.

\section{3]. The "BBF events"}

According to Angelopoulos et al. [6], the high-speed flows in the inner central plasma sheet (CPS) organize themselves in 10-min time scale flow enhancements called bursty-bulk flow (BBF) events. Both temporal and spatial effects are responsible for their bursty nature. The flow velocity exhibits peaks of very large amplitude with a characteristic time scale of the order of a minute, which are usually associated with magnetic field dipolarizations and ion temperature increases.

\section{4]. The review work of Sergeev at al. [7]}

According to the review work of Sergeev et al. [7], the substorm expansion and recovery phases are characterized by a complicated temporal structure both in the night-time plasma sheet and in the auroral ionosphere composed of multiple, elementary, local, sporadic and impulsive energy dissipation events (IDEs) having about 1-min duration. The characteristic signatures for these activations are highspeed flow bursts associated with magnetic flux transfer in the plasma sheet and the formation or activation of auroral arcs in the ionosphere. They identified the impulsive reconnection as the most likely mechanism for the IDEs; each IDE corresponds to a short-term dissipation process of a few $R_{E}$ in the plasma sheet or $\sim 1^{\circ}$ in latitude and 1 hour in MLT in the ionosphere. It is also assumed that the integrated effect of these activations form the substorm intensifications or multiple onsets $[8,9]$.

\section{5]. The PiB pulsations}

In general, in order to study the generation mechanisms of waves, several points should be studied, including (a) the frequency characteristics and harmonic structures; (b) the spatial distribution and possible propagation of the waves; (c) the polarization characteristics; (d) any possible correlation with IMF/solar wind parameters; (e) the correlation with geomagnetic activity, e.g., phases of storms and substorms; and (f) correlation with in situ particle data. All these issues are clearly discussed for the presented events in this work; we are interested in for waves directly related to substorms, and whose excitation mechanism is probably the recurring activations of the source, even in a monochromatic mode.

Geomagnetic pulsations, i.e., ultra-low-frequency (ULF) waves covering roughly the frequency range of the hypothesized pulsating twin-DL structure $(\mathrm{T}=15-50 \mathrm{~s})$ are the Pc3 ( $\mathrm{T}=10-45 \mathrm{~s})$ and Pc4 $(\mathrm{T}=45-150 \mathrm{~s})$ classes. These pulsations are studied in the past, with in situ observations in space using both magnetic and electric fields, and with ground-based magnetometers. Compressional Pc3 waves occur during daytime, having relatively low field amplitude fluctuations (a few nT) with typical periods of 20-30 s; most importantly, these waves correlate with the interplanetary magnetic field (IMF) and are thus most likely a direct result of compressional energy (derived from wave-particle interaction in the foreshock and shock region) impinging on the magnetopause and launching compressional fast mode waves to propagate through the magnetospheric cavity (e.g., $[10,11])$. In parallel, typical Pc4 pulsations are radially polarized transverse ULF waves localized close to the equator within a region which is radially very narrow, but longitudinally extending over several hours in the dusk sector (L being close to 6 at noon, and 8 at midnight). They are locally generated by freshly injected ions, but since the occurrence reaches its peak during a more quiet period after the substorms, some other mechanism is also affecting [11, 12]. Additionally, on rare occasions in the morning sector (about a few times a year), ground-based observations within the $\mathrm{Pc} 4$ range show highly monochromatic amplitude modulated oscillations, the so-termed "giant pulsations" [see, e.g., 13]. Therefore, we infer from all the already cited categories of ULF fluctuations that our events probably should be assigned to a different category. Our waves are (a) substorm-associated since they occur in PS with large tailward plasma velocities, and (b) characterized by very large $-\mathrm{B}_{\mathrm{z}}$ deviations which would be locally produced by the source or be carried away by the plasma flow. A $\Delta \mathrm{B}_{\mathrm{z}^{-}}$ decrease being approximately equal to the total $\mathrm{B}$, or as large as $20 \mathrm{nT}$ (in the near-Earth CPS), might be well-connected to local or neighboring CDs.

However, in contrast to the previously referred wave classes, $\mathrm{Pi} 2$ impulsive pulsations (in the period range from 40 to $150 \mathrm{~s}$ ) were recognized to be an integral part of the magnetospheric substorm. It is generally established that all of the members of the Pi2-class are generated by the onset of field-aligned currents and compressional waves in the nearEarth plasma sheet associated with substorm (look at the review of Olson [14], and references therein). The first process is the sudden generation of field-aligned currents in association with the disruption of cross-tail currents in PS and their subsequent effects on the ionosphere. The ionosphere appears to be something more than a passive load for this electrodynamic impulse. It responds sending currents back into a magnetosphere whose topology is changing and, perhaps, producing the feedback necessary to cause the explosive growth of the substorm current system. Oscillations of these currents are detected across the nightside of the Earth at onset of the mid-latitude and highlatitude portions of $\mathrm{Pi} 2$. After the impulse response of the inner magnetosphere to the $\mathrm{Pi} 2$ compressional waves, field line resonances, surface waves at the plasmapause and global oscillations are excited as well. With the onset of a substorm, a corresponding substorm current wedge is 
formed and begins to evolve, while oscillations in the current wedge field-aligned currents produce the mentioned high-latitude and mid-latitude $\mathrm{Pi} 2$ signals. The global occurrence of $\mathrm{Pi} 2$ pulsations is the single most important feature of these pulsations since it provides a diagnostic tool for substorm onset. However, it should be kept in mind that the occurrence is not simultaneous, nor is it actually global.
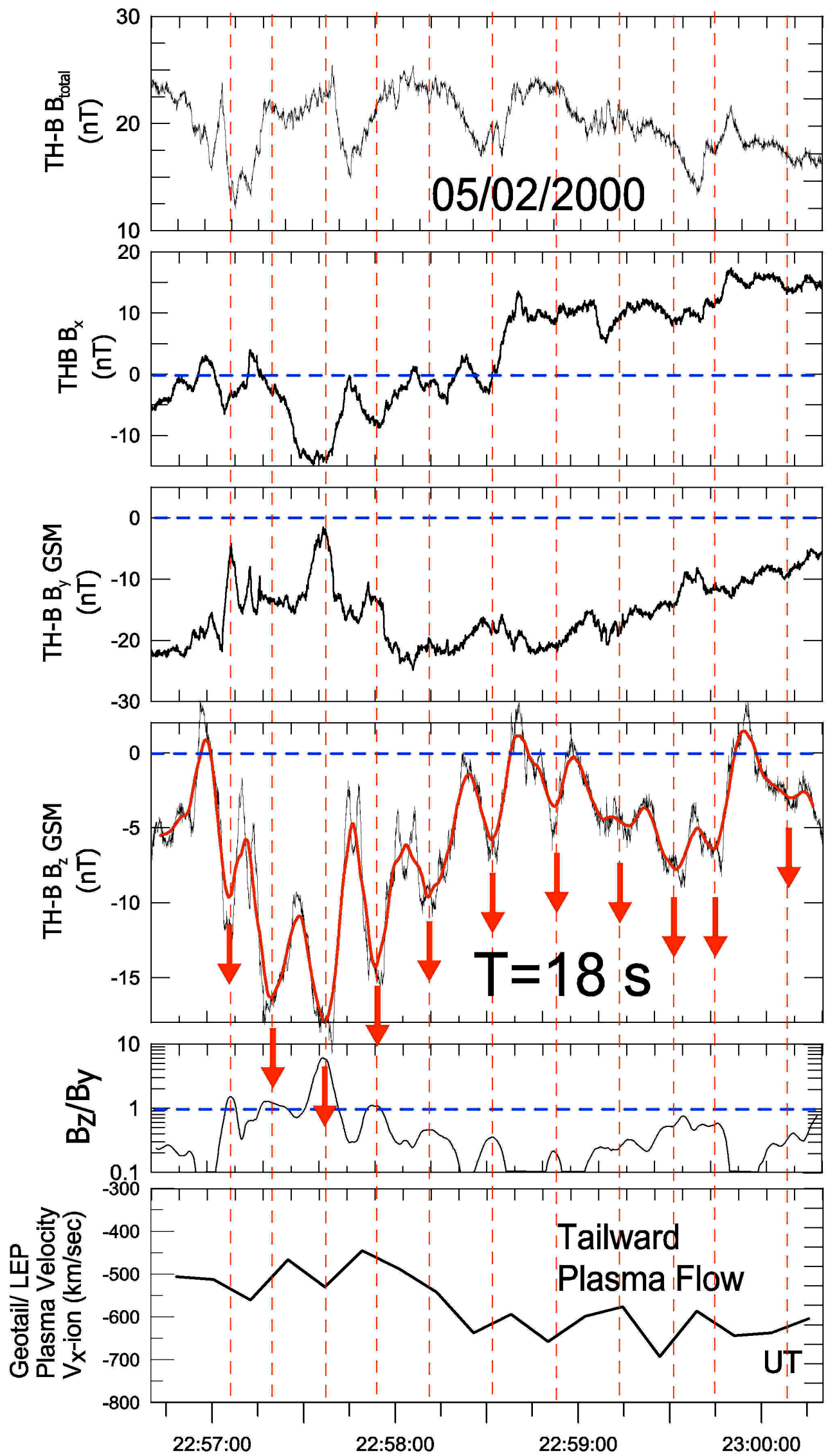

Fig. 1. Magnetic field components (in $\mathrm{nTs}$ ) along with the $\mathrm{V}_{\mathrm{x}}$ component of plasma velocity (in $\mathrm{km} \mathrm{s}^{-1}$ ) for an event occurred on $5 \mathrm{February}^{2000}$ The (marked with arrows) negative and quasi-periodic variations of $\mathrm{B}_{\mathrm{z}}$ are characterized by $\mathrm{T}=18 \mathrm{~s}$, while the plasma flows tailward. 
PiB pulsations are ULF, broadband, bursty geomagnetic pulsations occurring in the Pi1 $(\mathrm{T}=1-40 \mathrm{~s})$ and $\mathrm{Pi} 2$ frequency ranges. The $\mathrm{PiB}$ bursts are closely related to substorms $[15,16,17]$, and are thus good indicators of the substorm onset time. Because of the higher frequencies observed, the onset time can be defined more accurately using a PiB event than a more traditional Pi2 event. However, this is not always true, as the $\mathrm{Pi} 2$ pulsations extend to much larger geographical area than $\mathrm{PiB}$, and are thus easier to observe. In any case, if actually a pulsating source is the underlying physical process, then this fact by itself gives a reasonable answer to the excitation mechanism for the PiB-type pulsations.

In section 2, seven events observed by Geotail and potentially revealing a quasi-periodically pulsating source are studied in detail. The suggested model is analytically exhibited in section 3, wherein it is further discussed in the context of our observations and previously gained research knowledge in general.

\section{Observations}

We present seven events occurring in central plasma sheet (CPS), in which the $\mathrm{B}_{\mathrm{z}}$ component of the magnetic field demonstrates a quasi-periodic character with periodicity $\mathrm{T}=15-60$ s. In addition, for these events the plasma persistently flows tailward ( Tw); hence the corresponding component of velocity, $\mathrm{V}_{\mathrm{x}}$, has always negative sign. The presentation format is about the same for all the events. The vector magnetic field measurements (in nanoteslas), provided by the Geotail/MGF instrument [18] with 0.0625 sec resolution time, are shown along with the $\mathrm{V}_{\mathrm{x}}$ (in $\mathrm{Kms}^{-1}$ ). The Geotail plasma data are generated by sampling the moments over $12 \mathrm{sec}$ intervals of ion distribution functions measured by a low energy particle (LEP) instrument [19]. The $\mathrm{B}_{\mathrm{z}}$, on the average, remains negative, while its absolute value, $\left|B_{z}\right|$, at certain times, approaches the value of the total magnetic field, $\mathrm{B}_{\text {total }}$. In particular, we pave attention to the negative trace of $B_{z}$ conveying essential information concerning the source itself; the negative $B_{z}$ represents the most crucial parameter that underlines the strong contrast to the normal magnetotail topology always characterized by positive values.

\subsection{First Event on $05 / 02 / 2000$}

The satellite was positioned at $(\mathrm{X}, \mathrm{Y}, \mathrm{Z})_{\mathrm{GSE}}=(-30.1,-4.5$, 3) $R_{E}$, and ten variation cycles along the $B_{z}$ trace are clearly visible in Fig. 1 with a mean periodicity $T=18 \mathrm{~s}$. The $B_{z}$ trace is slightly smoothed (red line), although the highest resolution data are shown with a black-thin line, too. The average value of $B_{z}$ essentially remains negative, with a major value about $-20 \mathrm{nT}$. For this event, the $B_{y}$ persistently displays large values; thus we indicatively show the ratio $\mathrm{B}_{\mathrm{z}} / \mathrm{B}_{\mathrm{y}}$ (fifth panel) in order to highlight the relative significance of $B_{z}$. The bottom panel shows that the plasma always flows tailward with a velocity greater than $500 \mathrm{kms}^{-1}$; consequently, during this interval an activated source has to be at work. Finally, from this event, one could infer that an underline resonance mechanism may exist; the nature of the source dictates an almost monochromatic response. The $\mathrm{B}_{\mathrm{z}}$ fluctuations seem to be unaffected by the neutral sheet crossings, when the $\mathrm{B}_{\mathrm{x}}$ changes sign.

\subsection{Second Event on $04 / 04 / 2000$}

It occurred at $(X, Y, Z)=(-25.7,0,3.5) R_{E}$ (see Fig. 2), and is very similar to the preceded phenomenon; again, the $B_{z}$ shows about ten variation cycles although the periodicity range is broader, $\mathrm{T}=30-60 \mathrm{~s}$. The major negative value of $\mathrm{B}_{\mathrm{z}}$ is $-12 \mathrm{nT}$, the satellite was located well inside the PS and the plasma flows tailward with velocities higher than 700 $\mathrm{kms}^{-1}$ (fifth panel).
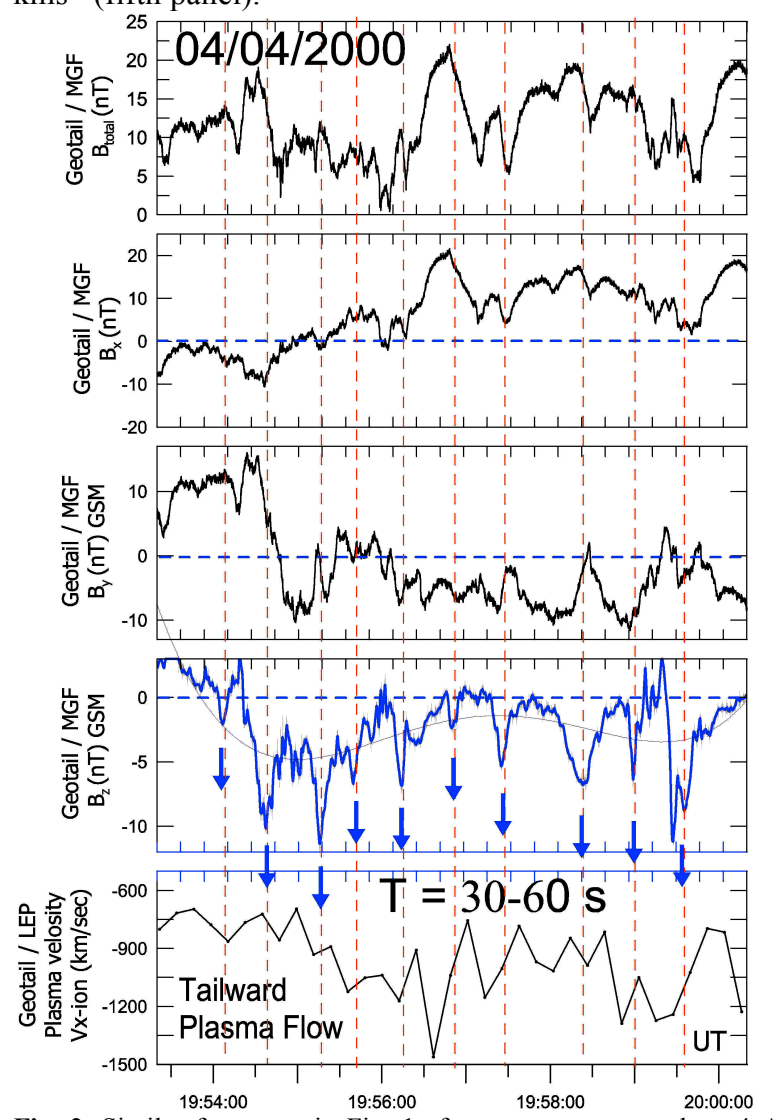

Fig. 2. Similar format as in Fig. 1 for an event occurred on 4 April 2000. The negative and quasi-periodic variations of $B_{z}$ are characterized by $\mathrm{T}=30-60 \mathrm{~s}$, while the plasma flows tailward.

\subsection{Third Event on $19 / 03 / 2000$}

This event occurred at $(-28.8,-0.2,2.5) \mathrm{R}_{\mathrm{E}}$ and gives two distinct clusters with $\mathrm{B}_{\mathrm{z}}$ fluctuations, which are shown as areas shaded with yellowish and bluish colors in Fig. 3. These areas are separated with an interval of relatively unvaried and high values of $\mathrm{B}_{\mathrm{z}}$; during this interval the satellite resides outside the CPS and closer to the PS boundary layer (PSBL), where $\mathrm{B}_{\mathrm{x}}>10 \mathrm{nT}$. An anticipated response, since the major magnetic field reconfigurations always take place within the CPS. The second group, of $B_{z}$ variations, is separately plotted at the bottom panel, where the band-pass filtered data with $\Delta \mathrm{T}=15-50 \mathrm{~s}$ are shown; the repetitive character of response is obvious. The $B_{z}$ variations are comparable, at times, to the $\mathrm{B}_{\text {total }}$, as the appropriate (sixth panel) reveals. Most importantly, the first cycles of $B_{z}$ variation occur while the tailward plasma velocity is $\sim 200$ $\mathrm{kms}^{-1}$ and the periodicity $\sim 50 \mathrm{~s}$; the next nine cycles in the second group occur with an average velocity $550 \mathrm{kms}^{-1}$ and a periodicity of $\sim 20 \mathrm{~s}$. Therefore, the supposed wavelength of variations essentially remains, for both cases, almost the same; that is, the plasma velocity affects the frequency according to the Doppler effect. A probable explanation is that the satellite goes closer to the source, and since the local PS cross-sectional area decreases around the source, the velocity increases. Consequently, for this event, the source- 
beat rather remains constant throughout the event; that is $\mathrm{T}=$ $20 \mathrm{~s}$ for the whole interval of $10 \mathrm{~min}$.

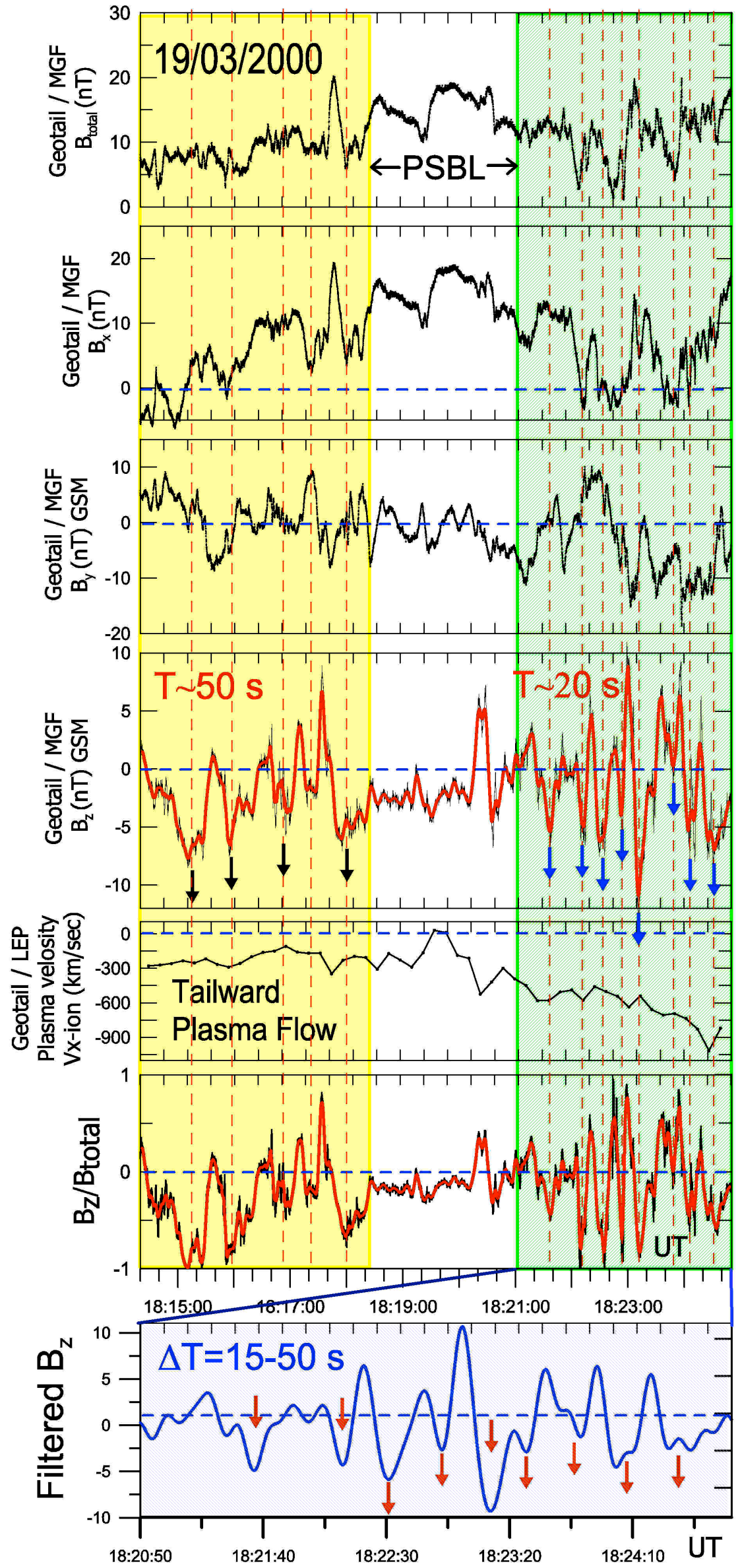

Fig. 3. Similar format as in Fig. 1 for an event recorded on 19 March 2000. The negative and quasi-periodic variations of $B_{z}$ with $T \cong 20 \mathrm{~s}$ occur with higher tailward plasma velocities than those with $\mathrm{T} \cong 50 \mathrm{~s}$. The amplitude of fluctuations is significant only in CPS. 


\subsection{Fourth Event on $02 / 03 / 2000$}

This event occurred at $(-30.1,0.9,2) \mathrm{R}_{\mathrm{E}}$, and one can identify two clusters with $B_{z}$ fluctuations (as in the preceded case) shaded with yellowish and bluish colors in Fig. 4. In between them, again, the satellite is temporarily removed, from the CPS, into the PSBL region, where apparently the "wave activity" is low. It should be underlined, that at certain times $\Delta \mathrm{B}_{\mathrm{z}} \cong \mathrm{B}_{\text {total }}$ (look at the bottom panel). The first four cycles are characterized by an average period $\mathrm{T}=45 \mathrm{~s}$, while the plasma flows tailward with velocity $\sim 600 \mathrm{kms}^{-1}$. During the second cluster, displaying more than ten variation cycles, $\mathrm{T}=18 \mathrm{~s}$ and $\mathrm{V}_{\mathrm{x}}=1400 \mathrm{kms}^{-1}$. Again, the wavelength seems to be the same (i.e., $\sim 4 \mathrm{R}_{\mathrm{E}}$ ); the change of frequency is probably due to the Doppler effect and the source is characterized by a constant rhythm with $\mathrm{T}=18 \mathrm{~s}$.
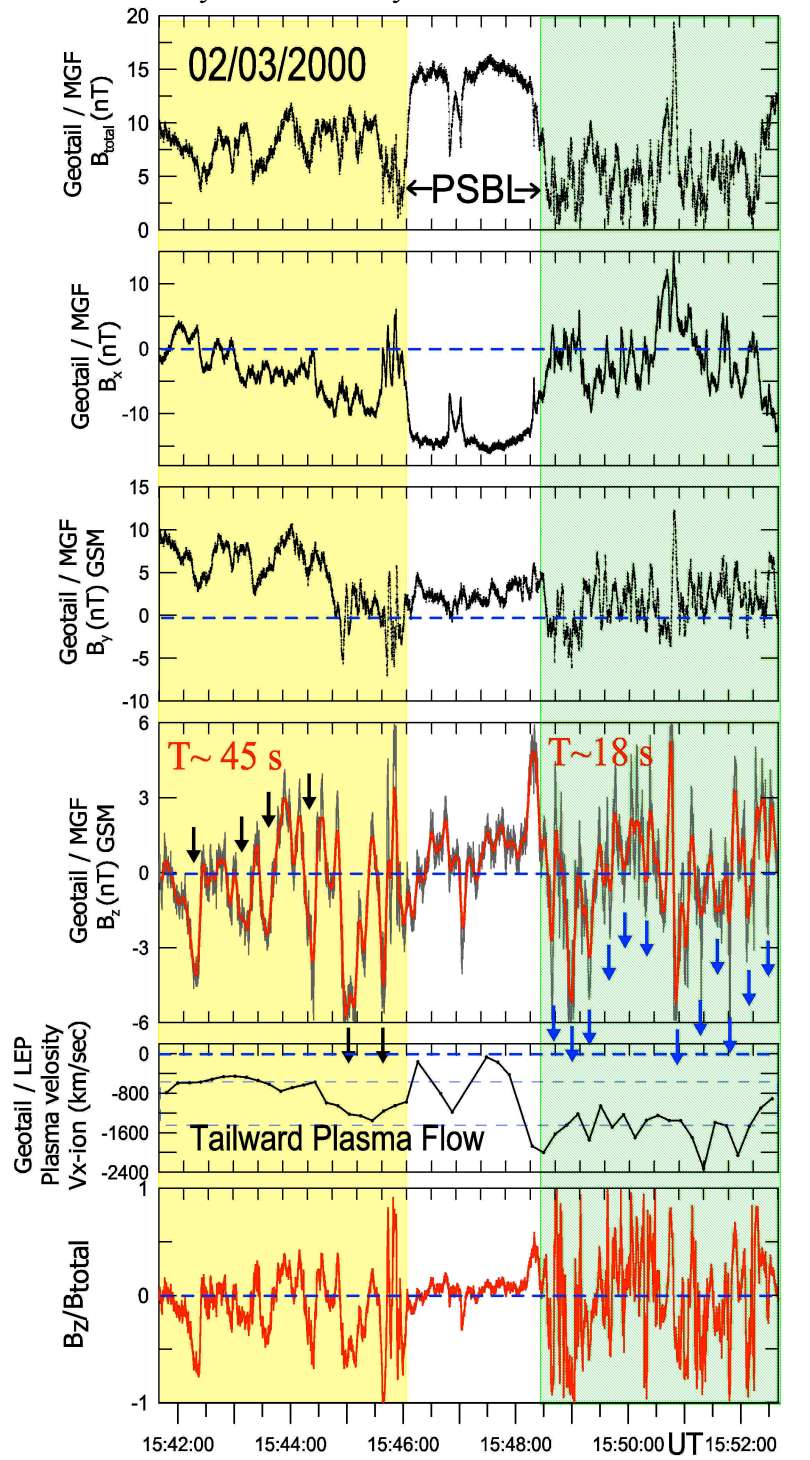

Fig. 4. Similar format as in Fig. 1 for an event recorded on 2 March 2000. The negative and quasi-periodic variations of $B_{z}$ with $T \cong 18 \mathrm{~s}$ occur with higher tailward plasma velocities than those with $\mathrm{T} \cong 45 \mathrm{~s}$; a result due to the Doppler effect. The amplitude of fluctuations is significant only in CPS.

\subsection{Fifth Event on 12/05/2005}

This event (plotted in Fig. 5) occurred at $(-29.2,7,2.7) R_{E}$ and, as in the preceded events, two groups of quasi-periodic $B_{z}$ variations are separated by an interval with lower wave activity when the satellite probably dwells close to the PSBL (i.e., $\mathrm{B}_{\mathrm{x}} \simeq-17 \mathrm{nT}$ ). The periodicity is $15-30 \mathrm{~s}$ and we can identify at least 15 repetition cycles. The $B_{z}$ fluctuations with more negative deflections are related to $\mathrm{V}_{\mathrm{x}}$-values exceeding $800 \mathrm{kms}^{-1}$; in addition, almost each $\mathrm{B}_{\mathrm{z}}$-variation corresponds to a large ratio of $\mathrm{B}_{\mathrm{z}} / \mathrm{B}_{\text {total }}$ (bottom panel).

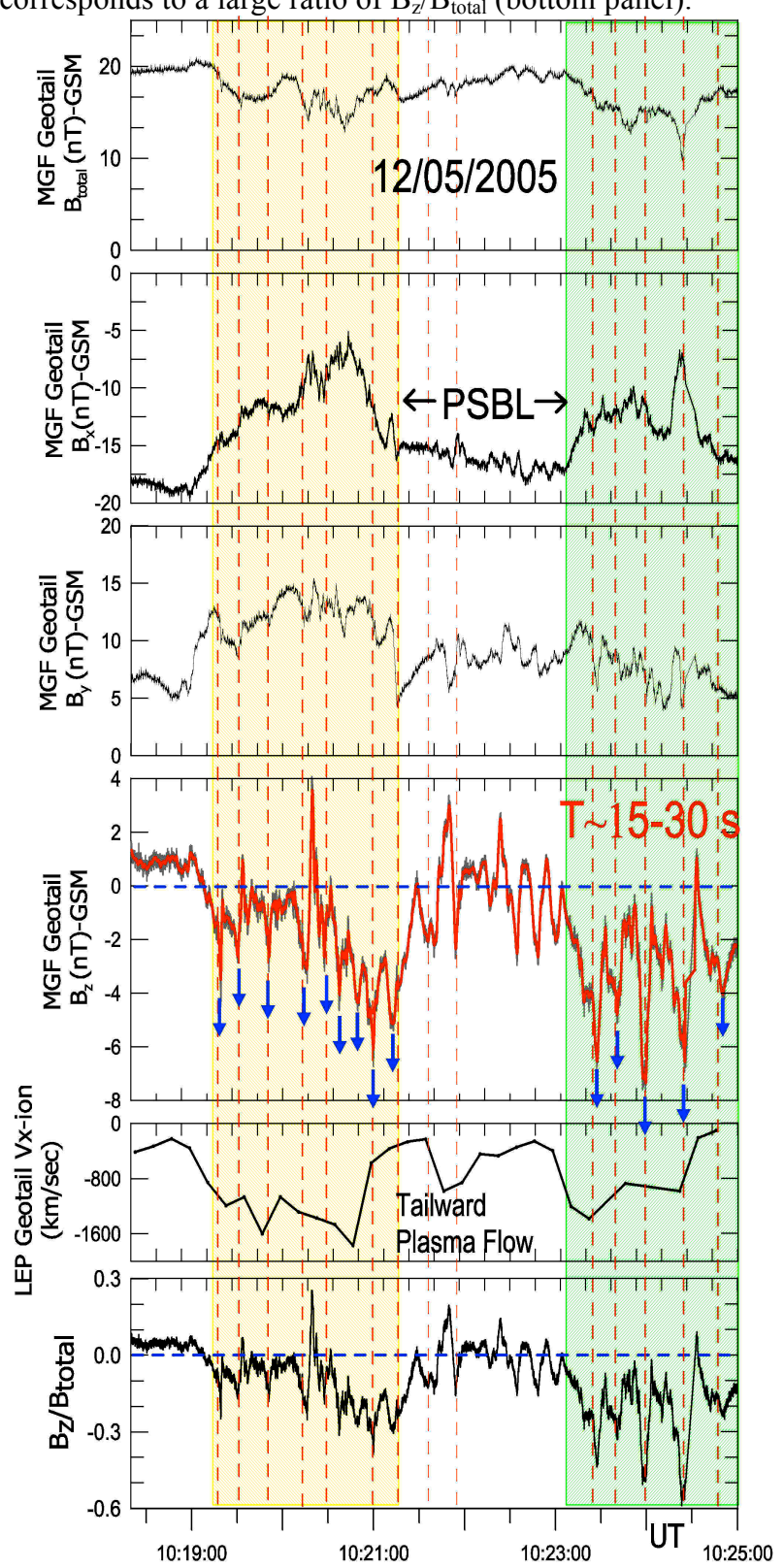

Fig. 5. Similar format as in Fig. 1 for an event recorded on 12 May 2005. The negative and quasi-periodic deflections of $B_{z}$ with $T \cong 15-30$ $\mathrm{s}$ occur only in CPS, with tailward plasma velocities.

\subsection{Sixth Event on 29/01/1998}

In this case study (plotted in Fig. 6) the satellite was located at $(-30.5,1.8,-2.1) \mathrm{R}_{\mathrm{E}}$, in CPS throughout the shown period, since the local lobe value is greater than $20 \mathrm{nT}$ (for this substorm). The negative $B_{z}$ fluctuations are profound and it is recommended to discriminate between the two marked groups of variations corresponding to extremely different velocities of plasma flow. In the first group of four cycles (marked with red arrows) the average $\mathrm{T}$ is $\sim 35 \mathrm{~s}$ and the tailward velocity $-450 \mathrm{kms}^{-1}$; in the second group of four cycles (marked with blue arrows) the $\mathrm{T}$ is $\sim 13 \mathrm{~s}$ and the 
velocity about $-1200 \mathrm{kms}^{-1}$. Consequently, the wavelength remains almost the same, $\lambda \cong 2.5 \mathrm{R}_{\mathrm{E}}$, for both intervals: $A$ result, again, probably due to the Doppler effect. In this situation the source seems to oscillate at $\mathrm{T}=13 \mathrm{~s}$.

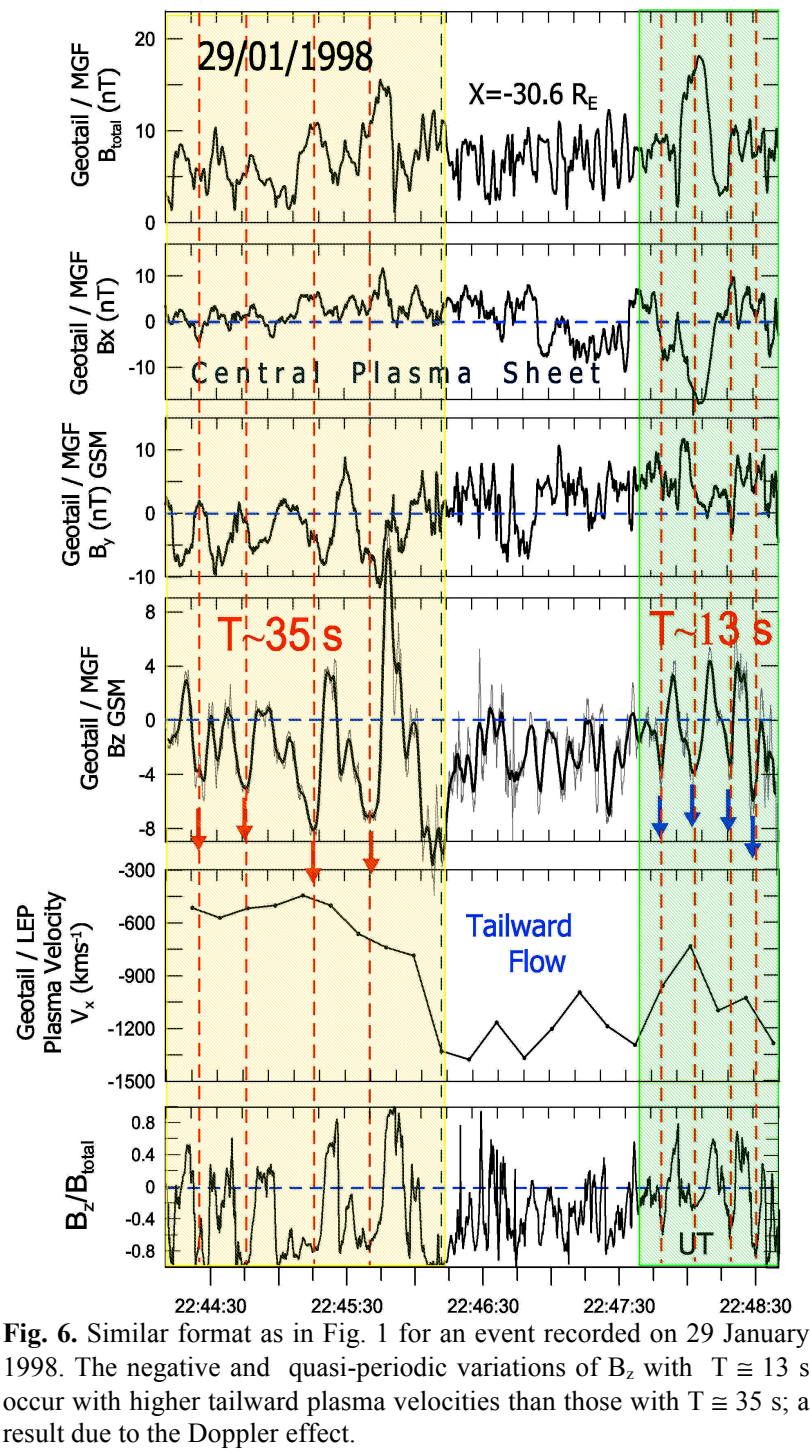

\subsection{Seventh Event on 08/02/1997}

In this case study the satellite was located at $(-28.7,10.1$, 0.4) $R_{E}$, staying within the CPS throughout the plotted period while the $\left|B_{x}\right|$ is always less than $10 \mathrm{nT}$ (Fig. 7). In contrast, the $B_{z}$ displays recurrent variations up to the extremely high value of $\sim 25 \mathrm{nT}$, with $\mathrm{B}_{\mathrm{z}} \cong \mathrm{B}_{\text {total }}$; the polar angle (theta, fourth panel) better demonstrates six variation cycles with large increases. The plasma flows tailward with a velocity $\sim 600 \mathrm{~km} / \mathrm{s}$. It is worth noticing that the periodicity is about $15 \mathrm{~s}$, and one can argue that this event probably shows six recurrent, rhythmic, current disruptions in CPS

\subsection{Conclusions}

The major conclusions inferred from the observations are as follows:

1]. While the plasma in magnetotail flows tailward, quasiperiodic variations along the $B_{z}$ trace with periodicities ranging from 15 to $60 \mathrm{~s}$ are frequently observed. In some cases we recognize almost a monochromatic response. The repetitive cycles of $B_{z}$ occur with southward turnings of the magnetic field. The latter by itself is a challenge in magnetotail's morphology and dynamics, where the normal topology (that is, the non-substorm magnetic field configuration) is always characterized by positive $B_{z}$ values.
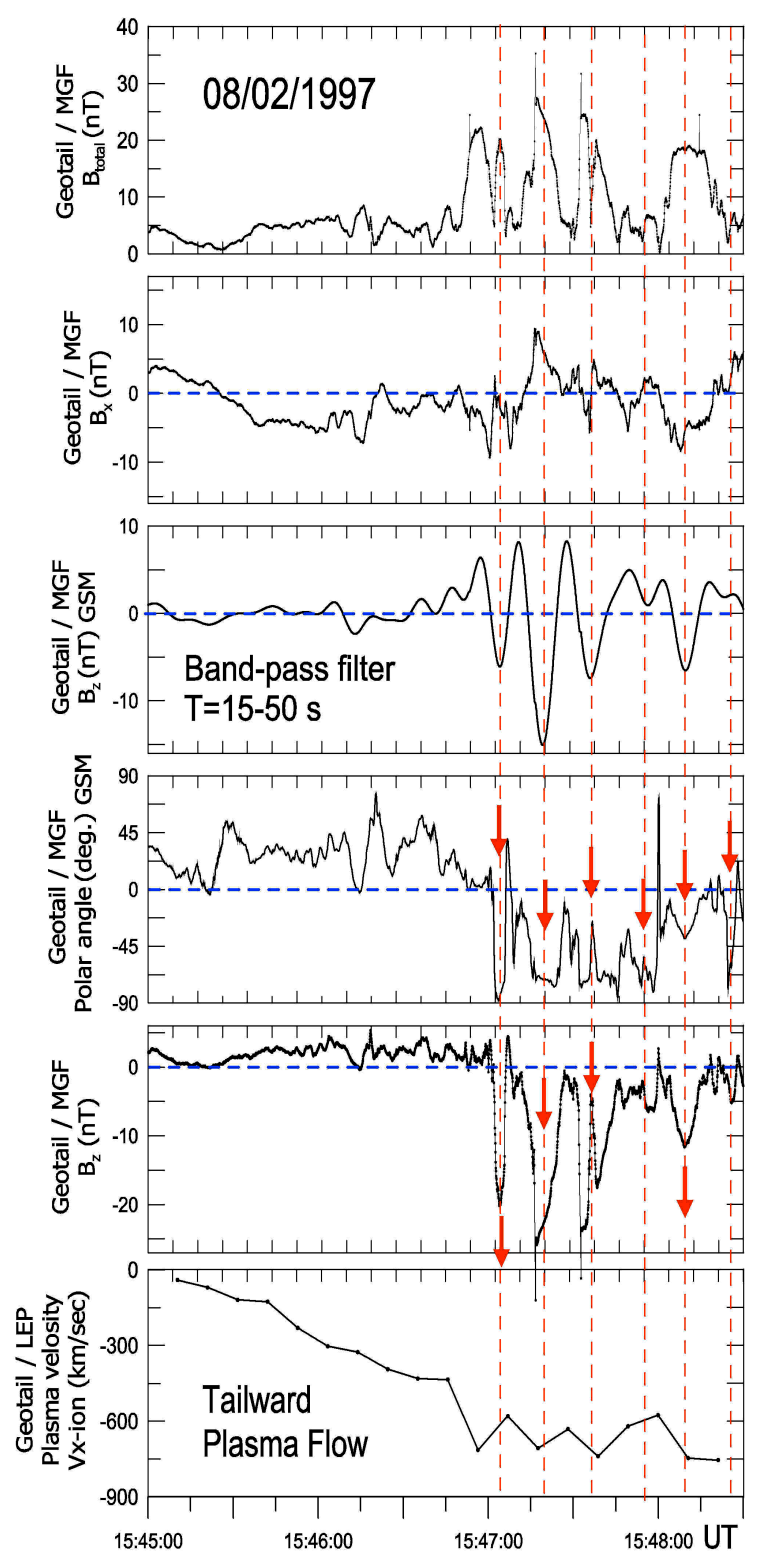

Fig. 7. Similar format as in Fig.1 for an event recorded on 8 February 1997. The very high amplitude, negative and quasi-periodic variations of $\mathrm{B}_{\mathrm{z}}$ with $\mathrm{T} \cong 15-50 \mathrm{~s}$ occur in CPS with high tailward plasma velocities.

2]. In other cases, we observe an impressive change in frequency which is probably due to the Doppler effect. The velocity enhancement increases the frequency, as well; thus, the higher velocity reveals better the real potential value of natural resonance for the source. It can be assumed that the higher velocities indicate proximity to the source.

3]. At certain times, the $B_{z}$ values are comparable to the local $\mathrm{B}_{\text {total }}$ and, additionally, the $\Delta \mathrm{B}_{\mathrm{z}}$ variations, locally, equal the amplitude of $\mathrm{B}$ at lobes. Therefore, one can plausibly argue that the $B_{z}$ variation cycles reflect the very dynamics of the source itself, and may be associated with abrupt changes or disruptions of the cross-tail current.

4]. The quasi-periodic wave activity of $\mathrm{B}_{\mathrm{z}}$ (occurred with tailward plasma flows) is much lower outside the CPS 
region. Each satellite entry into the CPS is commonly

\section{Discussion}

\subsection{The pulsating twin-DL structure}

We consider that the source place in magnetotail, wherein magnetic energy is converted to kinetic, is the pulsating twin-DL structure. The DL acts either as a resistor or a capacitor. The resistive behaviour is due to the fact that the DL impedes the movement of charges across it. The capacitive behaviour sets in when the DL is so thin that an accumulation of charged particles on one side develops an electrical force that pulls oppositely charged particles toward the other side. One may argue that the twin-DL structure behaves like a two-stroke (two-cycle) engine, which completes a power cycle with two strokes, comprising contraction and expansion movements of the whole structure. Certainly, beyond this figurative similarity, all the other procedures are extremely different. The contraction (compression) phase represents the rapid local thinning of PS that terminates explosively by heating plasma and accelerating energetic particles. The produced populations are finally ejected outward of the source along the outflow regions. The expansion (relaxation) phase corresponds to a local distention of the source which, in our case, also terminates explosively. The machine's intake phase, in which ions and electrons flow in toward the twin-DL region, extends behind the expansion phase with its final explosion. Alternatively, one may suggest that the twin-DL system pulsates in a way similar to the cardiac cycle with repeated, rhythmic contractions of heart. However, a fundamental difference to both of the above mentioned mechanisms is that in magnetotail the source retreats tailward throughout the substorm. This latter feature may remind us an analogous behavior encountered in a neuron cell whenever a signal-pulse transmission takes place along its axon; an "action potential" moves along the axon and temporally depolarizes the pre-existing polarization status of cell's membrane. Likewise in the twin-DL structure, the depolarization and re-polarization processes occur repetitively in successive cycles.

We hereafter term the expansion phase as "stage 1"; this is the phase driving the local source to its background or relaxation state. On the other side, the endpoint of this phase constitutes the starting point for the next "stage 2" of contraction. A detailed description for each stage is obviously necessary, if we are going to get an insight into more complicated issues like, for instance, the acceleration mechanisms of energetic particles in the magnetotail. We shall argue later on that the energetic electrons are accelerated at two distinct instants during each cycle: Tailward flowing electrons are always produced at the PS regions adjacent to the lobes (PSBLs) during the endpoint of contraction, whereas earthward flows are produced at the end of the expansion phase in CPS. This conclusion is in agreement with a previous work of Sarafopoulos [3] treating the just mentioned energetic electron bi-layer structure of counterstreaming particles.

\section{"Stage 1"}

The topology and dynamics of the twin-DL system was introduced by Sarafopoulos [2]. In that previous work the "stage 1" was introduced and exclusively described. In this present work, for the first time, we think about the "stage 2", accompanied by $\mathrm{B}_{\mathrm{z}}$ rhythmic variations.

which is particularly important in order to understand the source's repetitive cycles with recurring activations. We proceed to get an insight of "stage 1", in the "twin-DL" system, as follows:

First, in a thinned plasma sheet, where $r_{g i}$ and $R_{c}$ are the ion gyro-radius and the curvature radius of the local magnetic field, respectively, the chaotization of ion orbits begins when $R_{c}$ is about $9 r_{g i}$; while the full chaotization is achieved at the border case $R_{c}=r_{g i}[20]$, when the ion diffusion region (IDR) is formed. Certainly, the electrons under these conditions are not demagnetized; in contrast, they perform their adiabatic motion as previously. When $R_{c}$ $>9 \mathrm{r}_{\mathrm{gi}}$, the ion entry rate into the plasma sheet (over the meridional plane) is equal to the ion exit rate out of the plasma sheet. However, under the geometric condition $r_{g i} \leq$ $\mathrm{R}_{\mathrm{c}}<9 \mathrm{r}_{\mathrm{gi}}$, the ions largely lose their initial orbits and, consequently, slow down their velocities or even become temporally trapped within the thinned current sheet (TCS) region. Therefore, an ion accumulation process begins which builds up positive charge with potential serious consequences for the plasma sheet dynamics. The above geometric condition is rather frequently satisfied near the Earth and during magnetospheric substorms, when the plasma sheet thickness is measured to be $\sim 2000 \mathrm{~km}$ [21] and the $r_{\text {gi }}$ may have the typical value of $\sim 400 \mathrm{~km}$. In parallel, as the positive charge accumulates and the ion density locally increases, electrons will immediately flow neutralizing the additional positive charge. The surplus of positive (negative) charge is (from now on) denoted by $\mathrm{Q}^{+}$ $\left(\mathrm{Q}^{-}\right)$. Certainly, a portion from the $\mathrm{Q}^{+}$ions will be subject to pitch angle scattering and will finally precipitate into the ionosphere. During the $\mathrm{Q}^{+}$accumulation process, two layers with different plasma properties would be created at the two boundary regions (close to the northern and southern plasma sheet); therefore, two double layers (DLs) symmetric with respect to the neutral sheet are probably formed. We refer to the typical processes taking place in a plasma divided into two regions by a plane, wherein the one region has a higher electron density than the other side. In this situation, the electrons may freely stream in either direction and the flux of electrons from the dense plasma to the low density plasma will be greater than the flux of the electrons from the low density plasma to the dense plasma. Because many more electrons flow into the low density plasma than out of it, and the electron velocities are higher than the ion ones, part of the low density region becomes negatively charged. The dense plasma, conversely, becomes positively charged. Therefore, an electric field builds up, which starts to accelerate electrons towards the dense region, reducing the net flux. In this way, the electric field builds up until the fluxes of electrons in either direction are equal, and further charge build-up in the two plasmas is prevented; as a result, a DL is formed. In the Earth's magnetotail we shall have two DLs, one at each hemisphere, as it is schematically shown in Fig. 8a. Moreover, in this context, we can infer that the DL electric field is going to build up as far as new ions can enter into the central dense region; a demand that is satisfied when the ion thermal energy is higher than the DL potential drop $\left(\Phi_{\mathrm{DL}}\right)$. As soon as a balance is achieved, the potential drop will be equal to the ion thermal energy $\left(\mathrm{e} \Phi_{\mathrm{DL}} \approx \mathrm{k}_{\mathrm{B}} \mathrm{T}_{\mathrm{i}}\right)$; consequently, such a double layer is a marginally strong DL. In addition, we know that the ion 
thermal density in plasma sheet is about seven times the electron thermal density [22]. Finally, in a TCS, a twin-DL structure will be probably formed within the Earth's magnetotail; each DL is extended in the region where an electric field $\left(\mathrm{E}_{\mathrm{II}}\right)$ is oriented parallel to the magnetic field lines as drawn in Fig. 8a. Along the tail (i.e., the X-axis) the extent of an DL is roughly dictated by the condition $R_{c} \cong r_{\text {gi }}$. Within the sketched topology of symmetric DLs, the electron population that is initially accelerated in one DL will be equally decelerated in the other DL, after crossing the neutral sheet. Therefore, the electrons oscillate along the magnetic field lines and over the meridional plane; they obtain maximum (minimum) velocities at the neutral sheet (just at the outermost parts of their oscillation displacements). It should be pointed out that in the twin-DL structure, the overall charge of the ensemble is compensated, even though there are ambipolar electric fields parallel to the magnetic field lines.
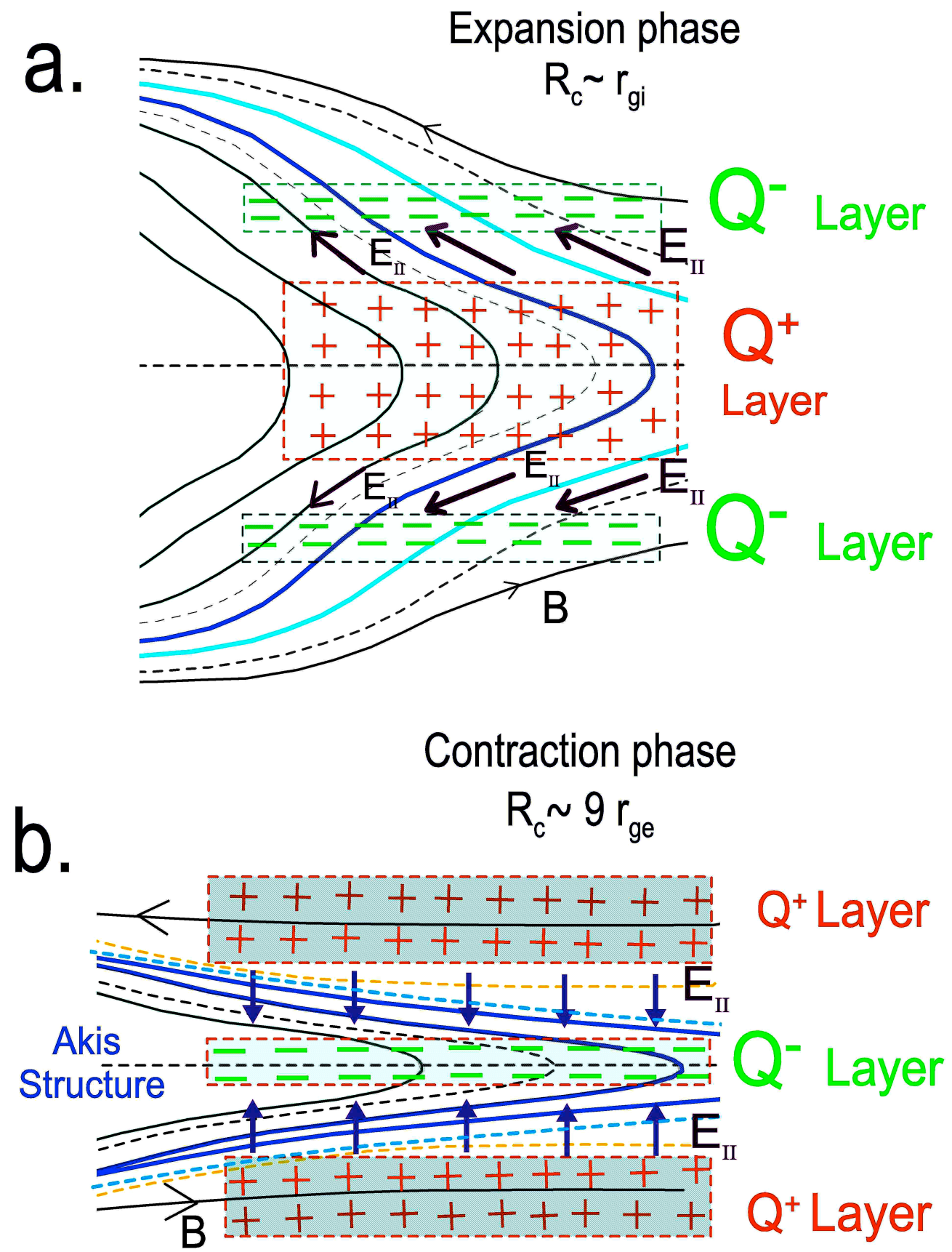

Fig. 8. Two distinct phases of the near-Earth PS corresponding to twin-DL structures with opposite polarities. The PS locally oscillates from the one phase to the other and vice versa, in many cycles repetitively. (a) The expansion phase $\left(R_{c} \cong r_{g i}\right)$ and (b) the contraction phase $\left(R_{c} \cong 9 r_{g e}\right)$ are essentially associated with different geometries and functions of the akis structure.

In "stage 1 " the PS seems to locally create a membrane region, the DL domain, in which a polarization electric field $E_{z}$ pointing outwards is developed. As a consequence of the $\mathrm{Q}^{+}$region formation, the twin-DL system should be associated with large scale Birkeland currents coupling the ionosphere with the distant activated centers in the 
magnetotail. The resulting gigantic electric resonant circuit with its inductance, capacity and resistance may eventually set the beat rate for the source itself

\section{"Stage 2"}

As the $R_{c}$ steadily decreases and becomes smaller than the ion gyroradius $r_{g i}$ and larger than a few electron gyro-radii $r_{\text {ge }}$ (i.e., indicatively $r_{\mathrm{gi}}>\mathrm{R}_{\mathrm{c}}>9 \mathrm{r}_{\mathrm{ge}}$ ), two major processes are noticeable: First, the "akis structure" (i.e., the sharp tip or the structure with extremely low $R_{c}$, is named according to the word akis coming from the ancient Greek work "áki $\boldsymbol{\zeta}^{\prime}$ indicating such a precious geometry) begins to play a new and exceptionally crucial role, since at the sharp tip region the ions are completely demagnetized and, most importantly, the local negative charge is redistributed. The electron density of akis continuously increases (as the $\boldsymbol{R}_{c}$ decreases) and the same does the associated electric field $E_{z}$. Finally, the $\mathrm{E}_{\mathrm{z}}$ of "stage 1" will reverse: The twin-DL structure is now strongly depolarized (fig. 8b). The akis corresponds to the $\mathrm{Q}^{-}$layer, which is surrounded by two $\mathrm{Q}^{+}$layers belonging to the IDR. In addition, the completely demagnetized ions (of the source) probably form two homoparallel cross-tail currents $\mathrm{J}_{\mathrm{y}}$ flowing adjacent to the lobes; that is, the single cross-tail ion current (flowing duskward in CPS) is now bifurcated into two distinct $\mathrm{J}_{\mathrm{y}}$ currents. The PS ions hitting the lobe magnetic field $B_{x}$ and performing their gyration motion come back into PS. Consequently, given that the two ion currents are mutually attracted, the whole structure will further reduce its size. Besides, the source electrons probably change dramatically their behavior: While they usually perform the $\mathbf{E}_{\mathrm{y}} \mathrm{x} \mathbf{B}_{\mathrm{x}}$ drift moving toward the neutral sheet and feeding the akis structure with negative charge, the situation now changes. As the inward directed electric field increases and becomes greater than the $E_{y}$, the PS electrons will essentially drift dawnward. Under this scenario, two new branches of electron currents would emerge flowing at a distance from the neutral sheet and in parallel to the $\mathrm{J}_{\mathrm{y}}$ ion currents.
Finally, as the $\mathrm{R}_{\mathrm{c}}$ monotonically decreases and all the currents are mutually attracted, the whole current system is forced to collapse. In this perspective, the smaller $R_{c}$ associated with the higher electron density (over the akis region) probably determines the main critical condition beyond which the collapse will be inevitable. And the condition will probably be the $\mathrm{R}_{\mathrm{c}} \approx 9 \mathrm{r}_{\mathrm{ge}}$, since after this limit the electrons initiate their demagnetization process. In front of akis, the same condition will set the maximum value for the earthward directed electric field. Probably beyond this limit the electron density does not suffice to further support the curl of the local magnetic field and the structure collapses. However, a better understanding of the collapse process will be revealed after incorporating the DL, as a distinct element, in the large magnetospheric circuit. And the collapse leads to a cross-tail current reduction or disruption or equivalently to an abrupt local dipolarization of the magnetic field. The ion and electron populations that remain within the twin-DL system must be redistributed within the new geometry; the $\mathbf{R}_{\mathbf{c}}$ rapidly increases and the source returns to its fundamental topology of "stage 1", the expansion phase. The instant of collapse is sketched in Fig. 9 , in which $B_{0}=B_{z o}$ is the value due to an unperturbed crosstail current, while the reduced source current $\mathrm{J}_{\mathrm{y}}$ is encircled by a (local) magnetic field perturbation $\triangle \mathrm{B}$ as Ampere's law dictates. Thus, a field value $\Delta B+B_{z 0}<0$ is developed at the tailward edge of the source, whereas a positive value of $B_{z}$ is developed at the earthward side (of the source). Therefore, the repetitively source activations will produce "negative pulses of $B_{z} "$, at the tailward side of the source; a process signifying the periodicity of the source itself. The collapse of the akis structure is related to the explosion phase, when an overvoltage is developed across each DL heating plasma and accelerating energetic particles. Then, the repolarization process starts in the twin-DL with the $E_{z}$ pointing outwards (and corresponding to the state of "stage $1 ")$.

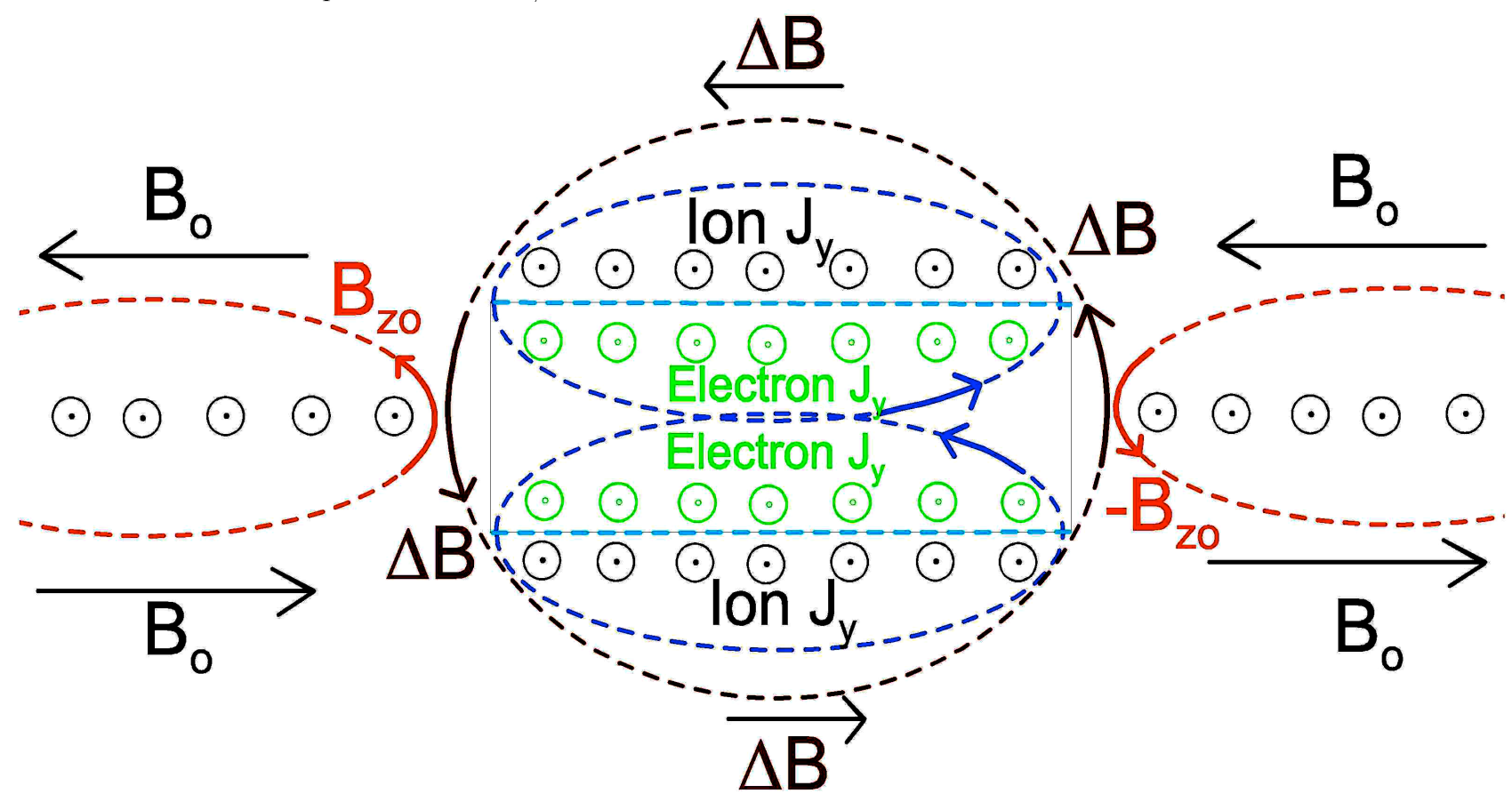

Fig. 9. At the instant of collapse, $B_{0}=B_{z o}$ is the magnetic field value due to the unperturbed cross-tail current, while the reduced current $J_{y}$ is encircled by a (local) magnetic field perturbation $\Delta \mathrm{B}$ as Ampere's law dictates. Thus, at the tailward edge of the source a field value $\Delta \mathrm{B}+\mathrm{B}_{\mathrm{zo}}<0$ is developed, whereas at the earthward side (of source) a positive value of $B_{z}$. Therefore, the repetitively source activations will produce "negative pulses of $B_{z}$ ", at the tailward side of the source; a process signifying the periodicity of the source itself. 


\subsection{The magnetotail/ionosphere RLC circuit and the twin- DL structure}

The Earth's night-side magnetosphere being a huge natural plasma laboratory in many aspects is similar to a simple circuit comprising an electrical generator (dynamo) in magnetotail, a gigantic current transmission line and a load over the ionosphere, especially at substorm times [23, 24]. It was already suggested that the twin-DL structure has probably the capability (a) to produce strong tailward ion jets during its explosive phase [2] and (b) to form ion vortices encircling strong $\boldsymbol{B}_{\boldsymbol{y}}$ cores like those observed (by Sarafopoulos [25]; for ropes tailward of the source) in MFRs embedded in tailward plasma flows. Moreover, in the latter work, it was demonstrated that the core's polarity of rope results as the joint action of the ion jet plus an upward or downward local motion of the tail: The upward motion corresponds to counter-clockwise circulation in vortex and consequently positive $\mathrm{B}_{\mathrm{y}}$ core, whereas the downward motion corresponds to clockwise circulation in vortex and consequently negative $\mathrm{B}_{\mathrm{y}}$ core. The differential motion between ions and electrons creates both an earthward directed electric field and a tailward directed current ([2]; also the same logic with a different mechanism was proposed by Lui and Kamide [26]). This is exactly the condition for a dynamo, where $\mathrm{J} \cdot \mathrm{E}<0$; the charge separation has been created with the breaking of the MHD condition. This dynamo process is certainly related to the meridional current system (MCS) for a substorm, which is the so-called Boström' s type II current system [27]. The meridional (i.e., mainly over the $\mathrm{XZ}$ plane) current passes through the night sector of the magnetotail and is composed of a pair of upward/downward field-aligned current (FAC) sheets plus the Pedersen ionosphere current.

We separately deal with four distinct snapshots (i.e., successive functional steps with, additionally, different topologies) of the whole system of the twin-DL structure associated with the ionosphere via the large scale Boström' s type II FACs. The steps are four because the circuit largely mimics the response of a resonating RLC circuit, in which the current reverses its direction within each cycle.

\section{a. Contraction Phase}

As the compression of the twin-DL structure proceeds the anticipated behaviour would be outlined as follows: The akis region (formed obviously in CPS, in close vicinity to the neutral sheet) steadily supplies the ionosphere circuit with negative charges. The reason for the latter is the progressively decreasing curvature radius $\left(R_{c}\right)$ of the magnetic field, which unavoidably is related to decrease of the charge $\mathrm{Q}^{-}$of akis; thus, electrons leave the structure. In parallel, as it is already stressed, the local electron density of akis increases and the same does the electric field $E_{z}$ in each DL. In general, we assume that there is an inverse proportionality between the electron density and the $R_{c}$. Moreover, the neutrality principle is satisfied within the volume of DLs, since the akis charge $\mathrm{Q}^{-}$is counterbalanced by $\mathrm{Q}^{+}$. Finally, current flows in the whole circuit of huge transmission line, all the way, from the DLs to the ionosphere; the circuit's load constitutes the ionosphere resistance, as it is shown in Fig. 10a. The inductance of line, the ionosphere resistance and the capacitance introduced by the DL, in a first approach, constitute an RLC circuit. The condition $9 \mathrm{r}_{\mathrm{ge}}<\mathrm{R}_{\mathrm{c}}<\mathrm{r}_{\text {gi }}$ is indicative for this variation phase.

\section{b. First current interruption in the ionosphere/magnetotail circuit}

The exhibited process in the preceded paragraph will abruptly halt, since the akis structure will collapse; at this very moment the cross-tail electron current does not suffice to support the curl of the magnetic field and the electrons of akis begin their demagnetization process. Therefore, this time the akis is unable to continue providing the needed current in the circuit; the current disrupts and an overvoltage $L \cdot d i / d t$ is produced across each DL, along with a very strong electric field (Fig. 10b) pointing outwards and accelerating particles. The field aligned component of the electric field, $\mathrm{E}_{\mathrm{II}}$, will accelerate electrons tailward, and a satellite $(\mathrm{S} / \mathrm{C})$ located in PSBL will clearly detect these energetic electrons streaming tailward; in contrast, in CPS the electrons will not show any clear flow direction. At the instant of current disruption, there is not any net charge over the akis or elsewhere within the twin-DL system; the involved geometry might be indicatively determined by the condition $\mathrm{R}_{\mathrm{c}} \approx 9 \mathrm{r}_{\text {ge }}$. Then, we step on to the next phase, in which fresh current is developed in the magnetosphere/ionosphere system.

\section{c. Current flowing to the opposite direction}

Immediately after the collapse of akis, the twin-DL structure will automatically expand since the cross-tail current is locally interrupted; thus, the $\mathrm{R}_{\mathrm{c}}$ becomes greater than $\mathrm{r}_{\mathrm{gi}}$, as the PS undergoes a dipolarization process. The $\mathrm{Q}^{+}$layer is now formed in CPS, while the $\mathrm{Q}^{-}$layer remains close to the PSBL; the whole structure looks like that shown in Fig. 8a. Consequently, several ions leak out of the twin DL system along the X-axis (Fig. 10c) and the resulting current, flowing in the external circuit, has reversed its flow direction as compared to that observed during the contraction phase (Fig. 10a); the DL electric field points outwards. Later, the continuing expansion inevitably will lead to the second current disruption described in the next phase.

\section{d. The second current interruption associated with the expansion phase}

During "step c", the circuit current (of Fig. 10c) stores magnetic energy in the inductor; then, the current naturally reaches to an endpoint, as the $R_{c}$ increases to a few $r_{g i}$. This moment all the ions become magnetized and no current flows through the circuit. The abrupt halt of current temporary produces an electric field $\mathrm{E}_{\mathrm{z}}$, as the circuit reacts in order to preserve the current flow; the $E_{z}$ is now inward directed (Fig. 10d), and the life of $E_{z}$ is depended on the time needed for the stored magnetic energy (in the element $\mathrm{L}$ ) to be released as kinetic energy. Energetic particles are accelerated by $E_{z}$, while the source begins to contract; however, it should be noted that the PS now is, locally, much thicker $\left(\mathrm{R}_{\mathrm{c}} \approx \mathrm{r}_{\mathrm{gi}}\right)$ as compared to the thickness of extreme contraction $\left(\mathrm{R}_{\mathrm{c}} \approx 9 \mathrm{r}_{\mathrm{ge}}\right)$. Therefore, in this phase the energetic electrons should stream earthward close to the PSBL, whereas in CPS the electrons will show a more isotropic angular distribution. 

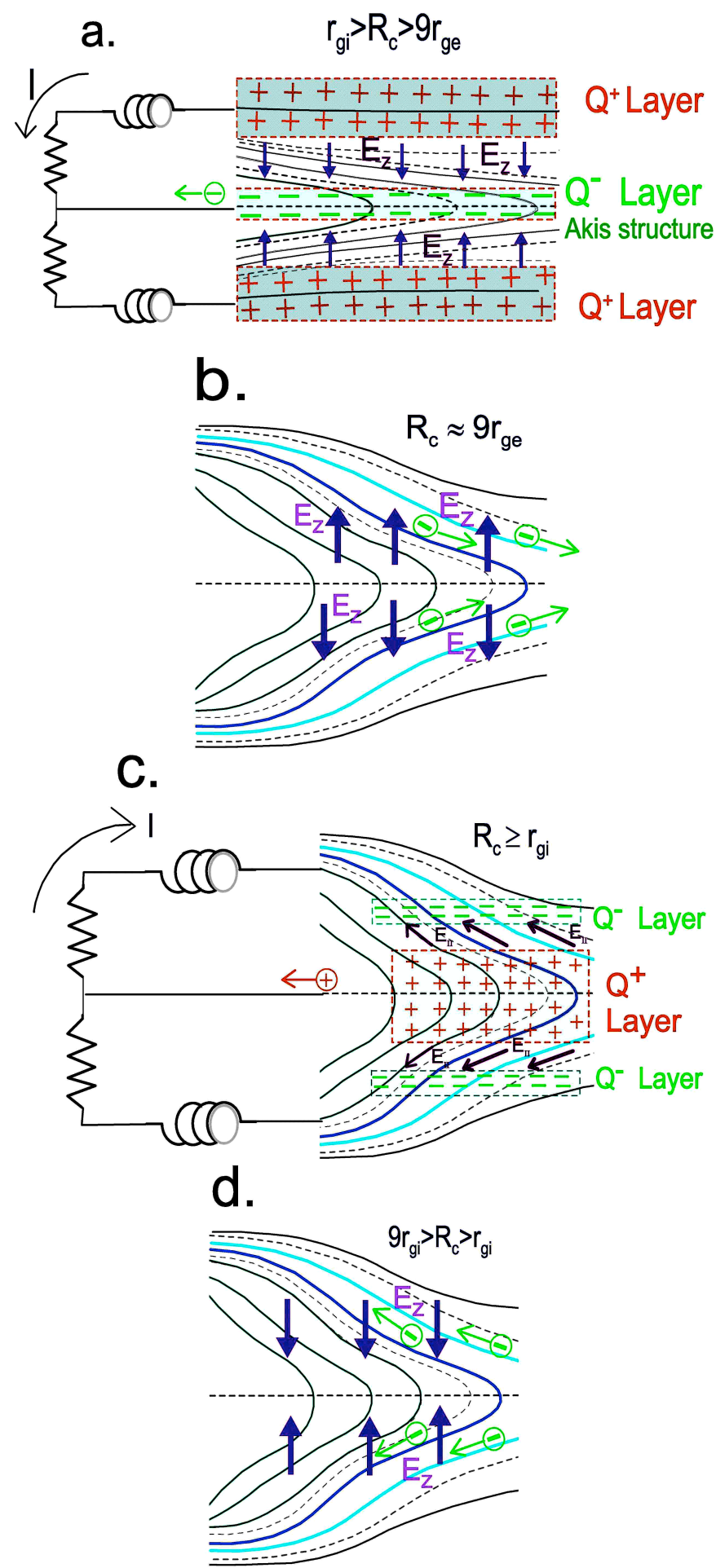

Fig. 10. The two distinct geometries of akis associated with the two opposite polarizations of the twin-DLs along with the large scale Boström' s type II FACs form a circuit that largely mimics the response of a resonating RLC circuit. The current reverses its direction within each cycle. At the phases (b) and (c) the current disrupts and an overvoltage is produced across each DL; at this very moments a very strong electric field pointing outwards or inward and accelerating particles is also developed.

\subsection{Model explaining the bi-layer structure of energetic electrons}

During the first current interruption (Fig. 10b), occurred in the ionosphere/magnetotail circuit and described in the preceded subsection, the energetic electrons flow tailward. In contrast, during the second current interruption (Fig. 10d) of the same circuit, the electrons stream earthward. Moreover, since (a) the satellite essentially remains stationary for time scales of a few minutes, (b) the twin-DL system expands and contracts with $\mathrm{T}=15-50 \mathrm{~s}$, and (c) the whole tail usually moves upward or downward with times larger than T, one can think as follows: First, on the basis of a pulsating source, during the phase of contraction, when the electrons always stream tailward, the satellite most likely will reside at the PSBL region. In contrary, during the phase of expansion, when the electrons stream earthward, the satellite most likely will reside in CPS. Moreover, on the basis of a continually moving tail, since the satellite temporally stays either at PSBL or in the CPS, it will recurrently detect either tailward or earthward fluxes of 
energetic electrons, respectively. If the satellite smoothly moves from PSBL to CPS (or vice versa), then the flow will reverse from tailward to earthward (or vice versa). The latter is what was very clearly inferred, on the basis of Geotail observations, in a published work by Sarafopoulos in 2010 [3]. He wrote "For the first time we identify a bi-layer structure of energetic electron fluxes in the Earth's magnetotail: Each bi-layer event is composed of two distinct layers with counterstreaming energetic electron fluxes, parallel and antiparallel to the local ambient magnetic field lines; in particular, the tailward directed fluxes always occur in a region adjacent to the lobes". He also noted that the NENL model is unable to produce the always tailward streaming electrons at the PS/lobe interface. Certainly, in the context of the twin-DL-pulsating structure, there is not any exclusively "spatial bi-layer structure" with streaming electrons. Instead, the bi-layer structure with antiparallel energetic electron fluxes is due to an oscillatory motion of the source itself, plus a systematically reversing electric field developed inside the source, for each variation cycle.

\subsection{The periodic current profile in the huge ionosphere/DL circuit}

In general, in an LC circuit the current in the inductor varies sinusoidaly, as it is plotted in Fig. 11 (upper panel); the resistance $\mathrm{R}$ is not presently taken into account. However, the introduction of a DL (instead of C) drastically modifies the above response of a simple harmonic oscillation. During the transition from instance 1 to 2 (as they are marked along the horizontal axis), when the twin-DL structure contracts (Fig. 10a), the current increases and sets up the magnetic energy in the inductor. At point 2, when the current reaches its maximum value $I_{0}$, all the energy is stored in the magnetic field: $\mathrm{U}_{\mathrm{B}}=\mathrm{LI}_{\mathrm{o}}{ }^{2} / 2$. In contrast, this moment the DL acting temporary as capacitor has no energy, which means $\mathrm{Q}=0$. Thus, $\mathrm{I}=\mathrm{I}_{\mathrm{o}}$ when $\mathrm{Q}=0$ (and $\mathrm{I}=0$ will be associated with
$\mathrm{Q}=\mathrm{Q}_{\mathrm{o}}$ ). Then, from point 2 to 3 , the current starts to charge the DL, whereas the current of the inductor decays; and it should be underlined that this moment the DL behaves radically different in comparison with the preceded stage: The DL acts much like a resistor and the current decreases as happens for the current in an RL circuit, $\mathrm{I}=\mathrm{I}_{0} \mathrm{e}^{-\mathrm{t} / \tau}$ with $\tau=\mathrm{L} / \mathrm{R}$, while $\mathrm{R}$ is the resistor of DL. That is, the current does not change sinusoidaly, as in an LC circuit wherein the capacitor begins to charge. In contrast, the electrostatic field within the DL, and particularly the field-aligned component of the electric field $\left(\mathrm{E}_{\mathrm{II}}\right)$, sets in motion the electrons so that the current changes exceptionally abruptly: The electrons in the neighborhood of neutral sheet rapidly become isotropic, whereas the electrons away from neutral sheet stream tailward. Finally, the current decays much faster and the $\mathrm{dI} / \mathrm{dt}$ change leads to an over-voltage across the DL. This moment the electrons and ions alike accelerate to high energies.

From point 3 to 4 , the source steadily expands, the curvature radius $\mathrm{R}_{\mathrm{c}}$ becomes comparable to the ion gyroradius $r_{g i}$ and, consequently, ions leak out from the twinDL system. Therefore, the circuit current has now the opposite direction (Fig. 10c) and the electric field of DL is directed outwards. This instant, the current again sets up the magnetic field and accumulates energy within the inductor $\mathrm{L}$. In turn, as the $\mathrm{R}_{\mathrm{c}}$ further increases, the ions become fully magnetized and the current in the circuit halts. The instant of $\mathrm{I}=-\mathrm{I}_{\mathrm{O}}$ signifies the appropriate time for the stored magnetic energy to be released. From point 4 to 5 (Fig. 11), the current continuous to flow to the same direction, the electric field is directed inward and, consequently, the electrons are basically accelerated earthward (Fig. 10d). Eventually, the circuit current rapidly decays and the source accomplishes a complete cycle. At the same time, the source begins to contract and a new cycle will be initiated

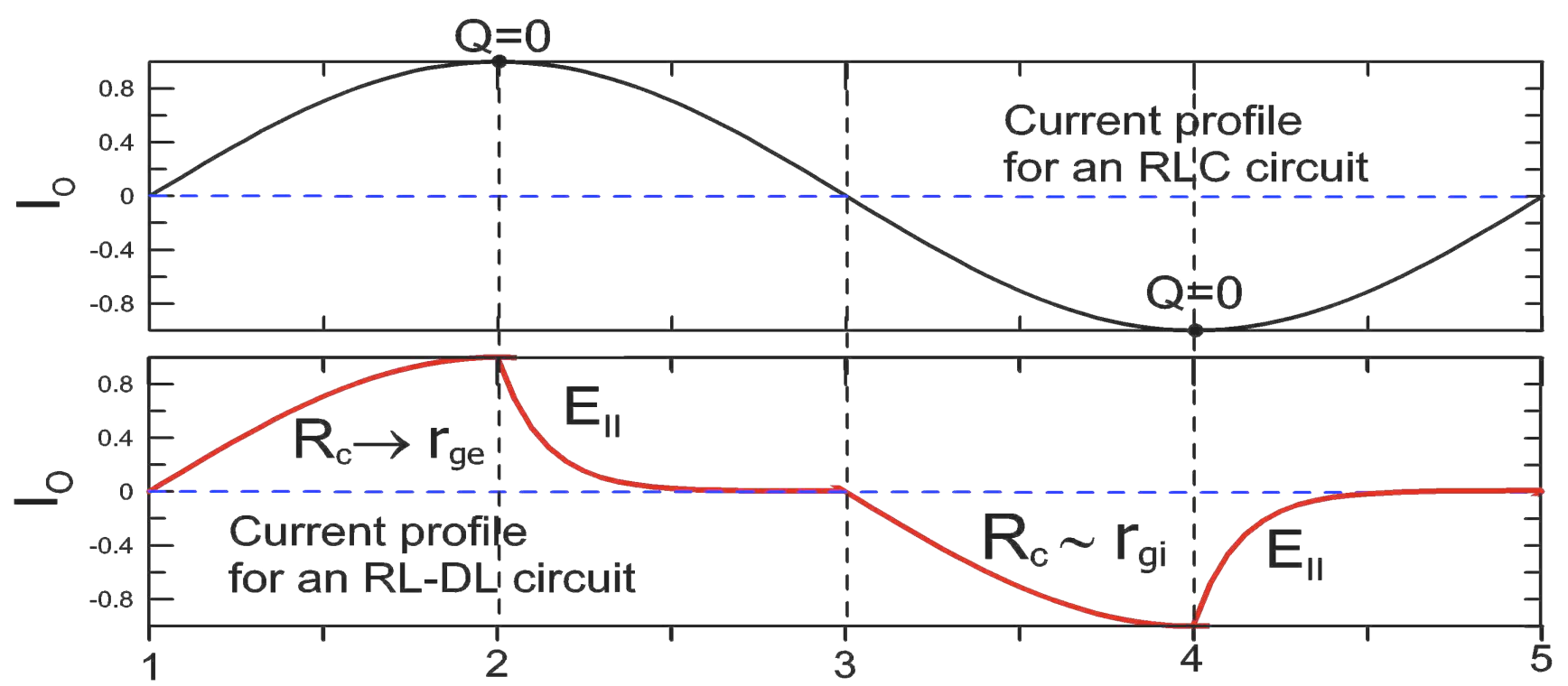

Fig. 11. In an LC circuit the current in the inductor varies sinusoidaly (upper panel); the resistance R is not taken into account. However, the introduction of a DL (instead of C) drastically modifies the above response: During the transition from instance 1 to 2 , when the twin-DL structure contracts, the current increases and sets up the magnetic energy in the inductor. From point 2 to 3 , the DL acts much like a resistor and the current decreases as happens for the current in an RL circuit.

\subsection{An impressive asymmetry between the expansion- and contraction-source phases}

It is very clear from the work of Sarafopoulos [3] introducing the bi-layer and counterstreaming electron fluxes within the PS, that the tailward streaming electrons are usually more collimated along the magnetic field lines and having higher fluxes (as compared to those of earthward flow). Thus, the tailward fluxes could result from a stronger accelerating electric field or by a bigger emf. And the latter could occur, in the context of this work, from a steeper 
variation of the $\mathrm{di} / \mathrm{dt}$ term during current disruption, in the whole RLC circuit; for a given L, the overvoltage within the $\mathrm{DL}$ is proportional to the $\mathrm{di} / \mathrm{dt}$. In conclusion, one can anticipate that the expansion phase must be significantly shorter than the contraction one. An issue that is already discussed by Sarafopoulos [1]; for instance, he indicatively showed, in his Fig. 13, that the contraction phase (termed there as local thinning) is usually 2-4 times longer than the expansion one.

\subsection{Self-similarity between the macroscopic and microscopic approach}

We presume that the small scale $B_{z}$ fluctuations are superimposed over the large scale $B_{z}$ variations, while both of them show similar signatures in different scales. During an isolated substorm, a satellite located in CPS, first detects the thinning or contracting phase (typically associated with negative $B_{z}$ values) and then the PS recovery phase (with positive $B_{z}$ values); it is assumed that the source moves tailward passing over the spacecraft. This is the macroscopic approach corresponding to a procedure that may last $\sim 15$ min. And the microscopic behavior (studied in this work) demonstrates a small-scale self-similar response: Each variation cycle, lasting $15-50 \mathrm{~s}$, is associated with a $-\mathrm{B}_{z}$ deflection followed by $\mathrm{a}+\mathrm{B}_{\mathrm{z}}$ one; both deflections are integrated in a cycle composed of its own contraction and expansion phases. That is, a lasting activation (i.e., a distinct substorm corresponding to one major cycle of $B_{z}$ ) is probably composed of a few tens of smaller activations with their own variation cycles of $\mathrm{B}_{\mathrm{z}}$. Consequently, near the Earth, the final $B_{z}$ trace will initially display successive and distinct dipolarizations and later a prevailing positive $B_{z}$ component.

\subsection{Physics of the Harang Discontinuity (HD)}

The Harang discontinuity is the locus of points in the nightside auroral zone across which the meridional component of the ionospheric electric field reverses from a basically poleward field on the equatorward side region to a basically equatorward field on the equatorward side.
Erickson et al. [28] proposed that the HD results from a dawn/dusk asymmetry in total energetic plasma content across the nightside of the magnetosphere: The deficit (surplus) of dawnside (duskside) cross-tail current must be compensated by upward currents from the ionosphere. These upward currents include the observed extension of upward region 1 and upward region 2 currents into what Iijima and Pøtemra call the "HD sector" [29].

In the framework of this work a different perspective for the physics of the HD might be adopted: We already have assumed that the Birkeland currents related to the Pedersen ionospheric currents, over a meridional plane, are directly associated with the twin-DL structure in magnetotail. However, in the preceded analysis, we have mainly focused on the temporal evolution of the source itself at a certain site, the satellite position. A somewhat different approach is also possible since both of the topologies, sketched in Fig. 8 and termed as contraction and expansion phases, probably coexist (the same time) at different positions along the Xaxis. We consider a locally thinned PS, wherein its earthward (tailward) segment satisfies the condition that the $\mathrm{R}_{\mathrm{c}}$ is only a few $\mathrm{r}_{\mathrm{gi}}\left(\mathrm{r}_{\mathrm{ge}}\right)$. Under this geometry, both polarization states (that was separately studied before) coexist at a given instant, and the $\mathrm{E}_{\mathrm{z}}$-inward (outward) pointing electric field is developed closer to the lobes (CPS), as it is shown in Fig. 12. The latter seems to line up with a result from a survey performed by Liang et al., [30] in the near-Earth plasma sheet. They studied cross-tail current disruption (CD) events and identified that "when the current sheet thickness is down to ion kinetic scales, the ions are demagnetized, and a quasi-electrostatic neutral sheetpointing electric field emerges (at the current sheet boundary) owing to the charge separation". Eventually, if the tail's electric fields of Fig. 12 are mapped on the ionosphere, then one would obtain the electric field configuration identified as HD. For the North Pole ionosphere, the negative $E_{z}$ of the tail maps to the equatorwad directed ionosphere electric field (associated with the westward auroral electrojet), while the positive $\mathrm{E}_{\mathrm{z}}$ of tail corresponds to the poleward directed ionosphere electric field.

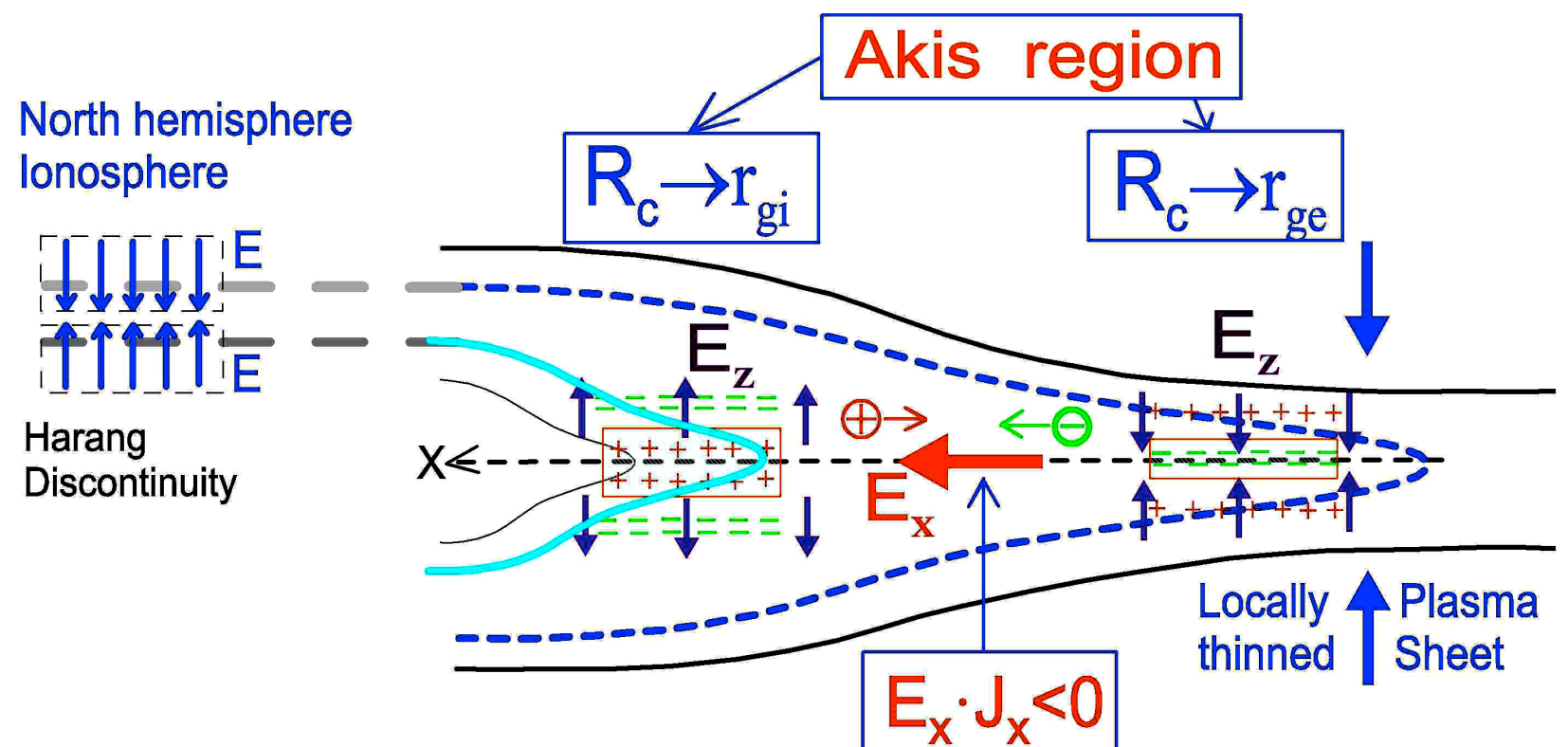

Fig. 12. Two distinct and oppositely polarized "twin-DL" structures occur in the near-Earth plasma sheet; they are coupled so that an inward directed electric field is developed between them. The left hand twin-DL (right hand twin-DL) loses ions (electrons) tailward (earthward), a result due to the formation of the ion diffusion region in the $r_{\text {gi }}$ scale (the akis effect in the $r_{\text {ge }}$ scale). The differential motion between ions and electrons creates both an earthward directed electric field and a tailward directed current, that is the condition for a dynamo, where $\mathrm{J} \cdot \mathrm{E}<0$. 


\subsection{The coupling of NENL and NECD}

From the perspective of Earth's magnetotail, there are two key phenomena in the evolution of a substorm: (1) initiation of the substorm current wedge (SCW) in the neargeosynchronous PS and (2) the onset of magnetic reconnection in the near-Earth to mid-tail PS. It is not surprising then that two basic models of substorm onset and expansion have been pursued: The synthesis near-Earth current disruption (NECD) and the near-Earth neutral line (NENL) models [e.g., 31, 32]. They are distinguished by whether or not reconnection in the near-Earth tail is a trigger or an event subsequent to substorm onset. For the NECD model, the initiation of the SCW results from onset of a nearby instability and disruption of cross-tail current.

In this work context, the "akis region" (sketched in Fig. 12) is composed of two distinct segments: The earthward part termed "left-hand twin-DL" or "region-A" which is characterized by the geometric condition of an $R_{c}$ approaching a few $r_{g i}$, and the tailward part termed "right hand twin-DL region or region-B" and characterized by an $\mathrm{R}_{\mathrm{c}}$ approaching a few $\mathrm{r}_{\mathrm{ge}}$. Moreover, one may assume that the region-A, although closer to the Earth, will reach earlier than the region- $B$ the required $\mathrm{R}_{\mathrm{c}} / \mathrm{r}_{\mathrm{ge}}$ relation leading to the phase of explosive dipolarization (or CD). In parallel, given that the ions always demagnetize earlier than the electrons, the ion flow could be observed tailward of either region-A or B, before any explosive phase [2]. The latter may suffice to explain why tailward flows are detected even before any ground based signature [e.g., 33, 34]. The sudden growth of the auroral electrojet will be essentially initiated with the explosive phase taking place in region-A (when $\mathrm{R}_{\mathrm{c}}$ tends to be a few $r_{g e}$ ). At the same very moment a sudden brightening of the auroral arc, which is located most equatorward in the oval, will take place as well.

In conclusion, one may say that the region-A (B) is associated with the specific features corresponding to the NECD (NENL) model.

\subsection{The internal dynamo process}

A key process proceeding from the akis structure, as we have just described it in the preceded paragraph, may be as follows: The region-A (B) loses ions (electrons) tailward (earthward), a result due to the formation of the ion diffusion region (the akis effect in the scale of $r_{g e}$ ). And the latter process may constitute the internal dynamo function that powers Boström' s type-II current system [27]. The surplus of positive (negative) charge to the right (left) leads to an earthward directed local electric field shown in Fig. 12, as it is anticipated by Akasofu (e.g., [35]). In our scenario, the differential motion between ions and electrons creates both an earthward directed electric field and a tailward directed current. This is exactly the condition for a dynamo where $\mathrm{J}$. $\mathrm{E}<0$, a result of charge separation created in the non-MHD fashion.

Lui and Kamide [26], proposed a dynamo for the meridional current system (MCS) to be a kinetic current disruption process such that dipolarization is achieved by magnetic field line slippage, thus producing the dynamo action of a tailward directed current with an earthward directed electric field. The electron convection speed associated with the field line slippage may be $\sim 150 \mathrm{~km} / \mathrm{s}$ [26], while the ion motion is considered to be largely unaffected by the field line slippage. However, in their scenario, one may argue that the magnetic field line slippage is oppositely directed during the tail' s "stretching" process, where the $R_{c}$ is less than $r_{g i}$. In contrast, in the akis structure the region-A and $\mathrm{B}$ are coupled forming an entity that will always exist in the near-Earth plasma sheet, and wherein the electric field will be earthward directed; as it is visualized in the snapshot of Fig. 12.

\section{Epilogue}

In the context of this work, each activation in the near Earth magnetotail is composed of many and frequently quasiperiodic micro-activations observed as recurring negative $B_{z}$ deflections in CPS. Moreover, we know that the dipolarization process in a substorm is most commonly considered as an indicator of current disruption; for a representative event very close to the Earth see the work of Takahashi et al., [5]. Thus in our model, each microdipolarization, for every variation cycle, is analogically manifested by a positive (or less negative) $B_{z}$ deflection. However, as a matter of fact, the source might be located more tailward and, then, the plasma flows would cause the dipolarizations. The latter led Birn and Hesse [36] to entitle their work "The current disruption myth". In parallel, one can argue that the reconnection idea, as a mechanism converting magnetic into kinetic energy and accelerating particles is a bigger myth, "a pseudo-science" as it was initially suggested by Alfvén [23, 24]. However, current disruptions (or reductions) and magnetic field reconfigurations associated with plasma flows are indeed detected in PS. This work explains, how the current disruption and, correspondingly, the magnetic field reconfiguration, essentially result as byproducts of a pulsating twin-DL structure. This author believed that Faraday's law can be experimentally validated more reliably in his suggested model, than in the alternative $X$ type magnetic reconnection model incorporating the concept of "magnetic diffusion". Under the X-type conditions, no one can be sure that the electron diffusion region is actually and persistently active; this would be an arbitrary assumption, and not a scientific insight based on facts. In contrast, it is our conviction that the "akis structure" is the most precious structure that could really play a unique role in redistributing charges and commutating the twin-DL system from depolarization to re-polarization and vise versa, for many repetition cycles. Whenever the $R_{c}$ approaches the distance a few $r_{g i}$, the akis forms the ion diffusion region. When the $\mathrm{R}_{\mathrm{c}}$ approaches the distance a few $r_{\text {ge }}$, at this very moment, the akis actually can support the needed surplus of negative charge (in CPS), finally leading to local current disruption and an overvoltage developed within each DL. When the $R_{c}$ decreases to a few $r_{g e}$, although the negative charge density of akis increases, however, the akis as a whole loses a portion of its charge supplying the external circuit with current. Large scale FACs flow and neutralize the net charges produced by "charge separation processes" within the twin-DL structure; and the latter naturally constitutes, under favorable conditions, a huge resonant RLC circuit. In this scheme, even the ionosphere probably plays its vital role initiating a positive feedback in the circuit. The proposed model and the related conclusions can be better validated if the whole system would be treated as a unique entity.

In conclusion, the quasi-periodic micro-activations observed near the Earth (as repetitive negative $B_{z}$ deflections) can be used as a diagnostic tool that potentially reveals the intra-source mechanism activating Faraday's law, 
which in turn heats plasma and accelerates particles. Additionally, it is clearly shown, in our section 2, that the source's repetition frequency is often changed by the Doppler effect; the frequency is affected by the plasma velocity. Therefore, it could be supposed that, at the source's site characterized by the thinnest PS, the velocity is probably maximized; in contrast, farther away from the source the frequency rather decreases. Finally, the pulsating DL structure is most probably characterized with the periodicity $\mathrm{T}=15-50 \mathrm{~s}$.

Certainly, the whole framework of this work is radically different from any "familiar, conventional and mainstream" consideration. The impartial reader, when adopting the presented physical model, has to take seriously into account one more fundamental thesis concerning the tail's dynamics: The author of this work suggested, and it is supported with in situ satellite observations [25], that well outside the source's region where the MHD theory is generally assumed to be valid, one can identify persistent ion jets. Moreover, these ion jets affected by local (upward or downward) magnetotail motions could result in large-scale ion vortices being the so-called "magnetic flux rope" topologies in magnetotail. Initially the streaming ions (close to the source and within the ion diffusion region) cross the magnetic field lines, whereas finally they become field-aligned forming the large scale "helical structure of rope"; while the sign of ion

\section{References}

1. Sarafopoulos, D. V.: A physical mechanism producing suprathermal populations and initiating substorms in the Earth's magnetotail, Ann. Geophysicae, 26, 1617-1639, 2008.

2. Sarafopoulos, D. V.: Twin-Double Layer Structure Producing Tailward Ion Jets in the Earth's Magnetosphere, Journal of Engineering Science and Technology Review, 5 (2), 76-84, 2012.

3. Sarafopoulos, D. V.: Bi-layer structure of counterstreaming energetic electron fluxes: a diagnostic tool of the acceleration mechanism in the Earth's magnetotail, Ann. Geophysicae, 28, 455-477, 2010 .

4. Sarafopoulos D. V. and E. T. Sarris.: Inverse Velocity Dispersion of Energetic Particle bursts inside the Plasma Sheet, Planetary and Space Science, 36, 1181-1199, 1988.

5. Takahashi, K., L. J. Zanetti, R. E. Lopez, R. W. McEntire, T. A. Potemra, and K. Yumoto.: Disruption of the magnetotail current sheet observed by AMPTE CCE, Geophys. Res. Lett., 14, 1019, 1987.

6. Angelopoulos, V., W. Baumjohann, C. F. Kennel, F. V. Coroniti, M. G. Kivelson, R. Pellat, R. J. Walker, H. Lühr, and G. Paschmann.: Bursty bulk flows in the inner central plasma sheet, J. Geophys. Res., 97(A4), 4027-4039, doi:10.1029/91JA02701, 1992.

7. Sergeev, V. A., T. I. Pulkkinen, and R. J. Pellinen.: Coupled-mode scenario for the magnetospheric dynamics, J. Geophys. Res., 101(A6), 13047-13065, doi:10.1029/95JA03192, 1996.

8. Yahnin, A. G., V. A. Sergeev, R. J. Pellinen, W. Baumjoharm, K. U. Kaila, H. Ranta, J. Kangas, and O. M. Raspopov.: Substorm time sequence and microstructure on 11 November 1976, J. Geophys., 53, 182, 1983.

9. Rostoker, G., S.-I. Akasofu, J. Foster, R. A. Greenwald, Y. Kamide, K. Kawasaki, A. T. Y. Lui, R. L. McPherron, and C. T. Russell.: Magnetospheric substorms- definitions and signatures, J. Geophys. Res., 85, 1663, 1980.

10. Engebretson, M. J., L. J. Cahill, Jr., R. L. Arnoldy, B. J. Anderson, T. J. Rosenberg, D. L. Carpenter, U. S. Inan, and R. H. Eather.: The role of ionosphere in coupling upstream ULF wave power into the dayside magnetosphere, J. Geophys. Res., 96, 1527-1542, 1991.

11. Anderson, B. J.: An overview of spacecraft observations of $10 \mathrm{~s}$ to $600 \mathrm{~s}$ period magnetic pulsations in the Earth's magnetosphere, in Solar Wind Sources of Magnetospheric Ultra-Low-Frequency Waves, eds. M. J. Engebretson, K. Takahashi, and M. Scholer, AGU Geophysical Monograph 81, 25-43, 1994.

12. Takahashi, K.: ULF waves: 1997 IAGA division 3 reporter review, Ann. Geophysicae, 16, 787-803, EGS - Springer-Verlag, 1998. circulation determines the core's polarity. The case of a "pseudo-magnetic flux rope" observed by the THEMIS satellites in the Earth's magnetotail is also very interesting. Strong but oppositely directed cross-tail magnetic field excursions, with positive " $\mathrm{B}_{\mathrm{y}}$ core" for TH-C and negative for TH-B, are almost simultaneously detected [37].

\section{Acknowledgements.}

We thank Prof. T. Nagai and Yoshifumi Saito for the high resolution Geotail/MGF magnetic field and Geotail/LEP plasma data, respectively. This research has been cofinanced by the European Union (European Social FundESF) and Greek national funds through the Operational Program "Education and Lifelong Learning" of the National Strategic Reference Framework (NSRF)-Research Funding Program: Thales. Investing in knowledge society through the European Social Fund. The project is called "Hellenic National Network for Space Weather Research" coded as MIS 377274. I thank the persons involved as reviewers.

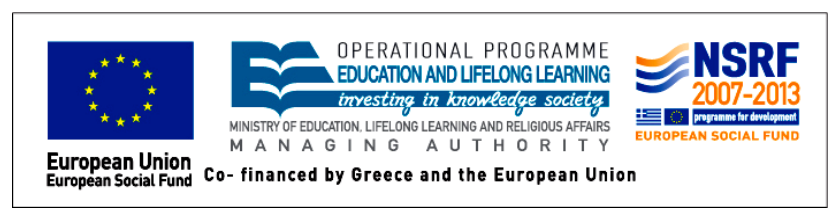

13. Rostoker, G., H.-L. Lam, and J. V. Olson.: Pc 4 giant pulsations in the morning sector, J. Geophys. Res., 84, 5153-5166, 1979.

14. Olson, J. V.: Pi2 pulsations and substorm onsets: A review, $J$. Geophys. Res., 104(A8), 17499-17520, doi:10.1029/1999JA900086, 1999.

15. Troitskaya, V. A.: Pulsations of the Earth's electromagnetic field with periods of 1 to 15 seconds and their connection with phenomena in the high atmosphere, J. Geophys. Res., 66, 5, 1961.

16. Campbell, W. H., and M. H. Rees.: A study of auroral coruscations, J. Geophys. Res., 66, 41, 1961

17. Bösinger, T., and A. G. Yahnin.: Pi1B type magnetic pulsations as a high time resolution monitor of substorm development, Ann. Geophysicae, 5, 231-238, 1987.

18. Kokubun, S., Yamamoto, T., Acuna, M. H., Hayashi, K., Shiokawa, K., Kawano, H.: The GEOTAIL magnetic field experiment, Journal of Geomagnetism and Geoelectricity, 46, 7-21, 1994.

19. Mukai, T., et al.: The low energy particle (LEP) experiment onboard the Geotail satellite. Journal of Geomagnetism and Geoelectricity, 46, 669-692, 1994.

20. Büchner, J. and Zelenyi, L.: Regular and Chaotic Charged Particle Motion in Magnetotail-like Field Reversals, 1. Basic Theory of Trapped Motion, J. Geophys. Res., 94(A9), 11821-11842, 1989.

21. Sarafopoulos, D. V.: Substorm - Associated Dual System of FieldAligned Currents Interpreting Major Bipolar Magnetic Field Signatures in the Earth's Magnetotail, Journal of Engineering Science and Technology Review, 6 (5), 149 -164, 2013.

22. Baumjohann, W., Paschmann, G., and Cattell, C. A.: Average plasma properties in the central plasma sheet, J. Geophys. Res., 94, 6597-6606, 1989.

23. Alfvén H.: Keynote address, NASA Conference Publication 2469, Proceedings of a workshop held at George C. Marshall Space Flight Center Huntsville, Alabama March 17-19, 1986a.

24. Alfvén H.: Double Layers and Circuits in Astrophysics, IEEE transactions on plasma science, Vol. PS-14, no. 6, p. 788, Dec. 1986b.

25. Sarafopoulos, D. V.: What does determine the sign of core in Magnetic Flux Rope structures of the Earth's magnetotail, Journal of Engineering Science and Technology Review, is accepted, 2014.

26. Lui, A. T. Y., and Y. Kamide.: A fresh perspective of the substorm current system and its dynamo, Geophys. Res. Lett., 30(18), 1958, doi:10.1029/2003GL017835, 2003.

27. Boström, R.: A model of the auroral electrojets, J. Geophys. Res., 69, 4983, 1964. 
28. Erickson, G. M., R. W. Spiro, and R. A. Wolf.: The physics of the Harang discontinuity, J. Geophys. Res., 96(A2), 1633-1645, doi:10.1029/90JA02344, 1991.

29. Iijima, T., and T. A. Potemra.: Large-scale characteristics of fieldaligned currents associated with substorms, J. Geophys. Res., 83(A2), 599-615, doi:10.1029/JA083iA02p00599, 1978.

30. Liang, J., W. W. Liu, and E. F. Donovan.: Ion temperature drop and quasi-electrostatic electric field at the current sheet boundary minutes prior to the local current disruption, J. Geophys. Res., 114, A10215, doi:10.1029/2009JA014357, 2009.

31. Lui, A. T. Y.: A synthesis of magnetospheric substorm models, $J$. Geophys. Res., 96, 1849, 1991

32. Baker, D. N., Pulkkinen, T. I., Angelopoulos, V., Baumjohann, W., and McPherron, R.L.: Neutral line model of substorms: Past results and present view, J. Geophys. Res., 101, 12,975-13010, 1996

33. Sergeev, V. A., V. Angelopoulos, D. G. Mitchell, and C. T. Russell.: In situ observations of magnetotail reconnection prior to onset of a small substorm, J. Geophys. Res., 100, 19121-19133, 1995.

34. Ohtani, S., F. Creutzberg, T. Mukai, H. Singer, A. T. Y. Lui, M. Nakamura, P. Prikryl, K. Yumoto, and G. Rostoker.: Substorm onset timing: The December 31, 1995, event, J. Geophys. Res., 104(A10), 22713-22727, doi: 10.1029/1999JA900209, 1999.

35. Akasofu, S.-I.: A source of auroral electrons and the magnetospheric substorm current systems, J. Geophys. Res., 108, 8006, doi:10.1029/2002JA009547, A4, 2003.

36. Birn J., and Hesse M.: The current disruption myth, Geophysical monograph 118 entitle Magnetospheric Current Systems, edited by S. Othani et al., 285-303, 2000.

37. Sarafopoulos, D. V.: A pseudo-magnetic flux rope observed by the THEMIS satellites in the Earth's magnetotail, Journal of Atmospheric and Solar-Terrestrial Physics, 73 (2011) 2279-2288, 2011. 